\title{
Critical Chirality in Elliptic Systems
}

\author{
Francesca Da Lio and Tristan Rivière*
}

October 22, 2020

\begin{abstract}
We establish the regularity in 2 dimension of $L^{2}$ solutions to critical elliptic systems in divergence form involving chirality operators of finite $W^{1,2}$-energy.
\end{abstract}

Keywords. Second order elliptic systems, Regularity, Integrability by compensation, Dirac operator, Quaternion algebra

MSC 2000. 35J47, 35B65, 34L40, 20G20

\section{Introduction}

In [14] the second author discovered a compensation phenomenon for the linear elliptic systems of the form

$$
-\Delta u=\Omega \cdot \nabla u \quad \text { in } D^{2}
$$

where $u \in W^{1,2}\left(D^{2}, \mathbb{R}^{n}\right), D^{2}=B^{2}(0,1)$ denotes the open unit ball centered at $(0,0)$ and $\Omega$ is an $L^{2}$ map into the antisymmetric matrices of $\mathbb{R}^{2}$ vectors. That is to say there exists a matrix $\left(\Omega_{i}^{j}\right)_{i, j=1 \cdots n}$ of $L^{2}$ functions into $\mathbb{R}^{2}$ such that

$$
\forall i=1 \cdots n \quad-\Delta u_{i}=\sum_{j=1}^{n} \Omega_{i}^{j} \cdot \nabla u_{j} \quad \text { and } \quad \Omega_{i}^{j}=-\Omega_{j}^{i} \quad \forall i, j=1 \cdots n .
$$

A-priori the system (I.1) is critical for the chosen norms, with a right hand side in $L^{1}$. Without the anti-symmetry of $\Omega$ no improved regularity has to be expected in general, while $W^{1,2}$ solutions to (I.1) for $\Omega \in L^{2}\left(D^{2}, \mathbb{R}^{2} \otimes s o(n)\right)$ are known to be in $\bigcap_{p<2} W_{l o c}^{2, p}\left(D^{2}\right)$.

One of the main strategy introduced in [14 was to use the antisymmetry of $\Omega$ in order to construct a "gauge" $A \in L^{\infty} \cap W^{1,2}\left(D^{2}, G l_{n}(\mathbb{R})\right)$ satisfying

$$
\operatorname{div}\left(\nabla_{\Omega} A\right):=\operatorname{div}(\nabla A-A \Omega)=0
$$

*Department of Mathematics, ETH Zentrum, CH-8093 Zürich, Switzerland. 
Taking a "primitive" $\nabla^{\perp} B=\left(-\partial_{x_{2}} B, \partial_{x_{1}} B\right):=\nabla_{\Omega} A \in L^{2}\left(D^{2}, M_{n}(\mathbb{R}) \otimes \mathbb{R}^{2}\right)$ the system (I.1) becomes equivalent to the conservation law

$$
\operatorname{div}(A \nabla u)=\nabla^{\perp} B \cdot \nabla u
$$

The Jacobian form of the right-hand-side of (I.2) permits to use now classical integrability by compensation phenomena originally discovered by H.Wente [22] and related to the ones by R.Coifman, Rochberg and Weiss [3] (see also [4]).

Following the main ideas of [14], extensions of this compensation phenomenon were obtained in [15] for critical systems of the form (for $m>2$ )

$$
\Delta v=\Omega v \quad \text { in } B^{m}
$$

where $B^{m}$ denote the $m$-dimensional ball centered at 0 and with radius $1, \Omega \in L^{m / 2}\left(B^{m}, s o(n)\right)$ and $v \in L^{m /(m-2)}\left(B^{m}, \mathbb{R}^{n}\right)$ as well as for systems of the form

$$
(-\Delta)^{1 / 4} v=\Omega v \quad \text { in } \mathbb{R}
$$

where this time $v \in L^{2}\left(\mathbb{R}, \mathbb{R}^{n}\right)$ and $\Omega \in L^{2}(\mathbb{R}, s o(n))$ (see [5]). More recently the two authors are extending their results to non local right-hand-side of the form

$$
(-\Delta)^{1 / 4} v=\int_{\mathbb{R}} H(x, y) v(y) d y
$$

where pointwise antisymmetry has to be replaced by the more general notion of antiself-duality of the underlying non-local operator $K(x, y)$ where $K(x, y):=H(x, y)-$ $\omega(x) \delta_{x=y} \in L_{l o c}^{1}\left(\mathbb{R}^{2}\right)$ (see [6]).

In the present work we are exhibiting a new compensation phenomenon which does not enter in none of the previous existing ones. Our main result is the following

Theorem I.1. Let $S \in \dot{W}^{1,2}\left(\mathbb{R}^{2}, O(n)\right)$, 1 such that $S^{2}=i d_{n}$ and let $u \in L^{2}\left(\mathbb{R}^{2}, \mathbb{R}^{n}\right)$ be a solution of the following linear elliptic system in divergence form

$$
\operatorname{div}(S \nabla u)=\sum_{j=1}^{n} \operatorname{div}\left(S_{i j} \nabla u^{j}\right)=\sum_{j=1}^{n} \sum_{\alpha=1}^{2} \frac{\partial}{\partial_{x_{\alpha}}}\left(S_{i j} u_{x_{\alpha}}^{j}\right)=0
$$

Then $u \in \bigcap_{p<2} W_{l o c}^{1, p}\left(\mathbb{R}^{2}, \mathbb{R}^{n}\right)$.

\footnotetext{
${ }^{1} O(n)$ denotes the group of orthogonal $n \times n$ matrices, $S O(n)$ is the group of orthogonal $n \times n$ matrices with determinant 1. $U(n)$ is the group of unitary $n \times n$ matrices and $S U(n)$ is the Lie group of $n \times n$ unitary matrices with determinant 1 . The Lie algebra of $U(n)$ consists of $n \times n$ skew-Hermitian matrices, with the Lie bracket given by the commutator.
} 
Remark I.1. The system (I.6) is elliptic with principal symbol $|\xi|^{2} S$. It is however not strongly elliptic in the sense of Legendre Hadamard 2 since obviously $\langle S \lambda, \lambda>$ can change sign as $\lambda$ varies.

Remark I.2. Structural conditions on $S$ for the regularity are necessary in the following sense. In [12] an $L^{2}$ solution to

$$
\operatorname{div}(A \nabla u)=0
$$

is produced where $A \in W^{1,2}\left(D^{2}, \operatorname{Sym}(2)\right)$ and $A$ is satisfying the strong ellipticity condition ${ }^{3}$

$$
<A(x) \xi, \xi>\simeq|\xi|^{2}
$$

uniformly on $D^{2}$ but $u \notin W_{l o c}^{1, p}\left(D^{2}, \mathbb{R}\right)$ for any $p>1$.

We also observe that we cannot expect in Theorem I.1 that $u \in W_{l o c}^{1,2}\left(D^{2}\right)$. Actually if we set $w=S u, w$ solves $\Delta w=\operatorname{div}(\nabla S S w)$. Such a PDE bootstraps in $W_{\text {loc }}^{1, p}\left(D^{2}\right)$ for $p<2$ but not in $W_{l o c}^{1,2}\left(D^{2}\right)$. If $w \in W_{l o c}^{1, p}\left(D^{2}\right)$ for $p<2$ then $w \in L^{p^{*}}\left(D^{2}\right)$ with $p^{*}=\frac{2 p}{2-p}$. By injecting such an information into the equation we get that $\nabla S S w \in L_{\text {loc }}^{p}\left(D^{2}\right)$ (since $\left.p=\frac{2 p^{*}}{p^{*}+2}\right)$ and therefore we come back to the initial information that $\nabla w \in L_{l o c}^{p}\left(D^{2}\right)$. This is not the case if $w \in W_{l o c}^{1,2}\left(D^{2}\right)$. This would actually imply that $w \in L_{l o c}^{q}\left(D^{2}\right)$ for every $q \geq 2$ and from the equation we deduce that $\nabla w \in L_{\text {loc }}^{\frac{2 q}{q+2}}$ which is a lost of information from the initial one since $1 \leq \frac{2 q}{q+2}<2$.

Remark I.3. Contrary to the case of the systems (I.1) in [14], we have not found yet striking applications in geometry or physics of systems (I.6) while nevertheless they look very "natural" and enjoy numerous formulations that we are going to present in this work. The system (I.6) is nothing but the Harmonic Map Equation into a pseudo-riemannian manifold (see remark I.4). The formulation using Dirac operator below (see I.10) moreover corresponds to the Weierstrass representation of Lagrangian surfaces in fourdimensional space by Hélein and Romon (11] Theorem 1). The assumption $u \in L^{2}$ is also

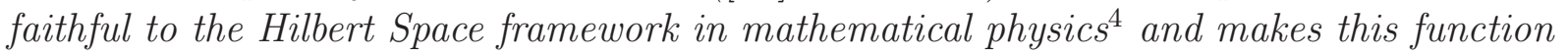
space natural in that sense.

\footnotetext{
${ }^{2}$ We recall that a matrix of coefficients $\left(A_{i j}^{\alpha, \beta}\right)_{1 \leq i, j \leq n}^{1 \leq \alpha, \beta \leq m}$ satisfies the strong ellipticity condition, or the Legendre-Hadamard condition if there exists $\lambda>0$ such that$$
A_{i j}^{\alpha, \beta} \xi_{\alpha} \xi_{\beta} \eta^{i} \eta^{j} \geq \lambda|\xi|^{2}|\eta|^{2}, \text { for all } \xi \in \mathbb{R}^{m}, \eta \in \mathbb{R}^{n} .
$$

In the case of the system (I.6) the matrix of coefficients is given by $A_{i j}^{\alpha, \beta}:=S_{i j} \delta_{\alpha, \beta}$ where $1 \leq \alpha, \beta \leq 2$, $1 \leq i, j \leq n$ and $\delta_{\alpha, \beta}$ denotes the Kronecker's operator.

${ }^{3}$ The matrix $A$ is acting on the different vertical components of $\nabla u, u$ is in fact scalar in this case while in theorem 1.1 the matrix $S$ acts on the horizontal components of $\nabla u$ that is $\nabla u_{1}, \cdots, \nabla u_{n}$.

${ }^{4}$ Original works in mathematical physics which have nourish the growth of analysis with problems from quantum mechanics, such as the study of Schrödinger semigroups for instance [19]...etc, take the $L^{2}$ space and not the "energy space" $W^{1,2}$ as the "configuration space".
} 
Behind the proof of theorem I.1 there is an $\epsilon$-regularity type of estimate which implies the following concentration-compactness result

Theorem I.2. Let $S_{k} \rightarrow S_{\infty}$ weakly in $\dot{W}^{1,2}\left(\mathbb{R}^{2}\right.$, Sym $\left.(n)\right)$ where $S_{k}^{2}=i d_{n}$ and let $u_{k} \rightarrow u_{\infty}$ weakly in $L^{2}\left(\mathbb{R}^{2}, \mathbb{R}^{n}\right)$ and satisfy

$$
\operatorname{div}\left(S_{k} \nabla u_{k}\right)=0 \quad \text { in } \mathcal{D}^{\prime}\left(\mathbb{R}^{2}\right) .
$$

Then, modulo extraction of a subsequence, there exists finitely many points $a_{1} \ldots a_{Q} \in \mathbb{R}^{2}$ s.t.

$$
u_{k} \longrightarrow u_{\infty} \quad \text { strongly in } \bigcap_{p<2} W_{l o c}^{1, p}\left(\mathbb{R}^{2} \backslash\left\{a_{1} \cdots a_{Q}\right\}\right) \text {. }
$$

Moreover $u_{\infty}$ satisfies $\operatorname{div}\left(S_{\infty} \nabla u_{\infty}\right)=0$ in $\mathcal{D}^{\prime}\left(\mathbb{R}^{2}\right)$.

We shall call $S \in \dot{W}^{1,2}\left(\mathbb{R}^{2}, \operatorname{Sym}(n)\right)$ where $S^{2}=i d_{n}$ a chirality operator. Etymologically, in old greek $\chi \varepsilon \iota \rho$ (kheir) means "hand". The word chirality refers to an intrinsic disymmetry of the space where a left and a right directions are given. More precisely almost everywhere on $\mathbb{R}^{2}$ we have the existence of two orthogonal projections, $P_{R}$ and $P_{L}$ complementary to each other $\left(P_{R}+P_{L}=i d_{n}\right)$, the left and the right, such that $S=P_{R}-P_{L}$.

Remark I.4. The system (I.6) is then the Euler-Lagrange equation of the Dirichlet energy into the pseudo-riemannian manifold $\left(\mathbb{R}^{n}, g\right)$ where

$$
g(X, Y):=\left\langle X, P_{R} Y\right\rangle-\left\langle X, P_{L} Y\right\rangle
$$

In other words (1.6) is the harmonic map equation from $\mathbb{R}^{2}$ into $\left(\mathbb{R}^{n}, g\right)$, it correspond to critical points of

$$
E_{g}(u):=\int_{\mathbb{R}^{2}}\left|P_{R} \nabla u\right|^{2}-\left|P_{L} \nabla u\right|^{2} d x^{2}
$$

As we will see theorem 1.1 can be rephrased as follows.

Theorem I.3. Let $P_{L} \in W^{1,2}\left(\mathbb{R}^{2}, \operatorname{Sym}(n)\right)$ such that $P_{L} \circ P_{L}=P_{L}$ and denote $P_{R}:=$ $i d_{n}-P_{L}$ and let $f \in L^{2}\left(\mathbb{R}^{2}, \mathbb{C}^{n}\right)$ satisfying

$$
\left\{\begin{array}{l}
P_{L} \frac{\partial f}{\partial z}=0 \\
P_{R} \frac{\partial f}{\partial \bar{z}}=0
\end{array}\right.
$$

then $f \in \bigcap_{p<2} W_{l o c}^{1, p}\left(\mathbb{R}^{2}, \mathbb{C}^{n}\right)$. 
In the course of the paper we will give a third formulation of our main result. For $n=2$ it takes a simpler following form.

Theorem I.4. Let $\Omega \in L^{2}\left(\mathbb{R}^{2}\right.$, so $\left.(2) \otimes \mathbb{C}\right)$ and let $f \in L^{2}\left(\mathbb{R}^{2}, \mathbb{C}\right)$ such that

$$
\frac{\partial f}{\partial z}=\Omega \bar{f}
$$

Assume $\Im\left(\partial_{\bar{z}} \Omega\right)=0$, then $f \in \bigcap_{p<2} W_{\text {loc }}^{1, p}\left(\mathbb{R}^{2}, \mathbb{C}^{2}\right)$.

The system

$$
\frac{\partial f}{\partial z}=\Omega f
$$

where $\Omega \in L^{2}\left(\mathbb{R}^{2}, s o(2) \otimes \mathbb{C}\right)$ and $\Im\left(\partial_{\bar{z}} \Omega\right)=0$ enjoys the same compensation property as (1.8) for $f \in L^{2}\left(\mathbb{R}^{2}, \mathbb{C}\right)$ but this last fact is a consequence of the theory in [14] while theorem $\Pi .4$ is new.

We will see that it can be recasted also in the following way. Recall first the definition of the Dirac Operator in $\mathbb{C}^{2}$

$$
\mathcal{D}:=\left(\begin{array}{cc}
0 & \partial_{z} \\
-\overline{\partial_{z}} & 0
\end{array}\right)
$$

Then we have the following corollary

Corollary I.1. Let $U \in L^{2}\left(\mathbb{R}^{2}, \mathbb{C}\right)$ such that $\Im\left(\partial_{\bar{z}} U\right)=0$. Let $\Psi \in L^{2}\left(\mathbb{R}^{2}, \mathbb{C}^{2}\right)$ be a solution of

$$
\mathcal{D} \Psi=\left(\begin{array}{cc}
U & 0 \\
0 & \bar{U}
\end{array}\right) \Psi,
$$

then $\Psi \in \bigcap_{p<2} W_{l o c}^{1, p}\left(\mathbb{R}^{2}, \mathbb{C}^{2}\right)$.

Throughout the paper we identify $\mathbb{R}^{2}$ with the complex number plane $\mathbb{C}$ and we will use both notations.

We will denote by $\mathcal{S}\left(\mathbb{R}^{2}\right)$ the space of Schwarz functions and by $\mathcal{S}^{\prime}\left(\mathbb{R}^{2}\right)$ the space of tempered distributions. For $1<p<+\infty$ we will denote by $\dot{W}^{1, p}\left(\mathbb{R}^{2}\right)$ the homogeneous Sobolev space defined as the space of $f \in L_{l o c}^{1}\left(\mathbb{R}^{n}\right)$ such that $\nabla f \in L^{p}\left(\mathbb{R}^{2}\right)$ and by $\dot{W}^{-1, p^{\prime}}\left(\mathbb{R}^{2}\right)$ the corresponding dual space $\left(p^{\prime}\right.$ is the conjugate of $\left.p\right)$.

We also denote by $L^{2, \infty}\left(\mathbb{R}^{2}\right)$ the space of measurable functions $f$ such that

$$
\sup _{\lambda>0} \lambda\left|\left\{x \in \mathbb{R}^{2}:|f(x)| \geq \lambda\right\}\right|^{1 / 2}<+\infty,
$$

and $L^{2,1}\left(\mathbb{R}^{2}\right)$ is the space of measurable functions satisfying

$$
\int_{0}^{+\infty}\left|\left\{x \in \mathbb{R}^{2}:|f(x)| \geq \lambda\right\}\right|^{1 / 2} d \lambda<+\infty .
$$


The spaces $L^{2, \infty}\left(\mathbb{R}^{n}\right)$ and $L^{2,1}\left(\mathbb{R}^{n}\right)$ belongs to the family of Lorentz spaces and one can check that they form a duality pair. For a nice introduction of Lorentz spaces we refer to [9].

In the sequel we will often use the symbols $a \lesssim b$ and $a \simeq b$ instead of $a \leq C b$ and $C^{1} a \leq b \leq C b$, whenever the constants appearing in the estimates are not relevant for the computations and therefore they are omitted.

Acknowledgments : A large part of the present work has been conceived while the two authors were visiting the Institute for Advanced Studies in Princeton. They are very grateful to the IAS for the hospitality. The authors are also very grateful to the anonymous referee and to Jerome Wettstein for useful remarks that permit us to improve the presentation of the paper.

\section{Preliminaries}

\section{II.1 Bourgain-Brezis Inequalities}

In [2] Bourgain and Brezis proved the following striking result:

Theorem II.5 (Lemma 1 in [2]). Let $u$ be a $2 \pi$-periodic function in $\mathbb{R}^{n}$ such that $\int_{\mathbb{R}^{n}} u=$ 0 , and let $\nabla u=f+g$, where $f \in \dot{W}^{-1, \frac{n}{n-1}}\left(\mathbb{R}^{n}\right)$ and $g \in L^{1}\left(\mathbb{R}^{n}\right)$ are $2 \pi$-periodic vector valued functions. Then

$$
\|u\|_{L^{\frac{n}{n-1}\left(\mathbb{R}^{n}\right)}} \leq c\left(\|f\|_{\dot{W}^{-1, \frac{n}{n-1}\left(\mathbb{R}^{n}\right)}}+\|g\|_{L^{1}\left(\mathbb{R}^{n}\right)}\right) .
$$

As a consequence of Theorem II.5 they get the following

Corollary II.2 (Theorem 1 in [2]). For every $2 \pi$-periodic function $h \in L^{n}\left(\mathbb{R}^{n}\right)$ with $\int_{R^{n}} h=0$ there exists a $2 \pi$-periodic $v \in W^{1, n} \cap L^{\infty}\left(\mathbb{R}^{n}\right)$ satisfying

$$
\operatorname{div} v=h \quad \text { in } \mathbb{R}^{n}
$$

and

$$
\|v\|_{L^{\infty}\left(\mathbb{R}^{n}\right)}+\|v\|_{W^{1, n}\left(\mathbb{R}^{n}\right)} \leq C(n)\|h\|_{L^{n}\left(\mathbb{R}^{n}\right)} .
$$

\section{II.2 Bourgain-Brezis inequality in 2 dimension revisited}

For the convenience of the reader we provide a proof of (II.11) in 2-dimension which has the advantage of not assuming periodicity. The proof is related to some compensation phenomena observed first in [7] in the analysis of 2-dimensional perfect incompressible fluids. This observation has also been used by the second author in the analysis of isothermic surfaces [16] (see also [8, 13, 14]).

We start by showing the following preliminary Lemma. 
Lemma II.1. Let $g \in L^{1}\left(\mathbb{C}, \mathbb{R}^{2}\right), f=\left(f_{1}, f_{2}\right) \in \dot{W}^{-1,2}\left(\mathbb{C}, \mathbb{R}^{2}\right)$ and $u \in \mathcal{S}^{\prime}(\mathbb{C}, \mathbb{R})$ be such that

$$
\nabla u=f+g \in\left(\dot{W}^{-1,2}+L^{1}\right)(\mathbb{C}) \quad \text { in } \mathcal{S}^{\prime}(\mathbb{C})
$$

Then there is $c \in \mathbb{R}$ such that $u-c \in L^{2, \infty}(\mathbb{C})$ and

$$
\|u-c\|_{L^{2, \infty}(\mathbb{C})} \leq C\left(\|f\|_{\dot{W}^{-1,2}(\mathbb{C})}+\|g\|_{L^{1}(\mathbb{C})}\right) .
$$

Proof of Lemma II.1. By assumption there exist $a_{j}^{k} \in L^{2}(\mathbb{C}, \mathbb{R})$ such that

$$
f_{j}=\sum_{k=1}^{2} \partial_{x_{k}} a_{j}^{k} .
$$

For $k=1,2$ we set $a^{k}=\left(a_{1}^{k}, a_{2}^{k}\right)$. Hodge decomposition in $L^{2}$ gives the existence of $\alpha^{k}, \beta^{k} \in \dot{W}^{1,2}(\mathbb{C})$ such that

$$
a^{k}=\nabla \alpha^{k}+\nabla^{\perp} \beta^{k}
$$

and

5 We have

$$
\left\|\nabla \alpha^{k}\right\|_{L^{2}}+\left\|\nabla \beta^{k}\right\|_{L^{2}} \leq\left\|a^{k}\right\|_{L^{2}}
$$

$$
\left\{\begin{array}{l}
\partial_{x_{1}} u-\sum_{k=1}^{2} \partial_{x_{k}} a_{1}^{k}=g_{1} \\
\partial_{x_{2}} u-\sum_{k=1}^{2} \partial_{x_{k}} a_{2}^{k}=g_{2}
\end{array}\right.
$$

We observe that

$$
\begin{aligned}
& \partial_{x_{1}} a_{1}^{1}=\partial_{x_{1}}\left(\partial_{x_{1}} \alpha^{1}-\partial_{x_{2}} \beta^{1}\right) \\
& \partial_{x_{2}} a_{1}^{2}=\partial_{x_{2}}\left(\partial_{x_{1}} \alpha^{2}-\partial_{x_{2}} \beta^{2}\right) \\
& \partial_{x_{1}} a_{2}^{1}=\partial_{x_{1}}\left(\partial_{x_{2}} \alpha^{1}+\partial_{x_{1}} \beta^{1}\right) \\
& \partial_{x_{2}} a_{2}^{2}=\partial_{x_{2}}\left(\partial_{x_{2}} \alpha^{2}+\partial_{x_{1}} \beta^{2}\right) .
\end{aligned}
$$

${ }^{5}$ One can show (II.14 by using the Fourier transform and the theory of Fourier symbols associated to a differential operator. If we denote by $\mathcal{F}\left[a^{k}\right]$ the Fourier transform of $a^{k}$ we have

$$
\begin{aligned}
\mathcal{F}\left[\partial_{x_{1}}(-\Delta)^{-1}\left(\operatorname{div} a^{k}\right)\right] & =-i \xi_{i}|\xi|^{-2}\left(i \xi_{1} \mathcal{F}\left[a_{1}^{k}\right]+i \xi_{2} \mathcal{F}\left[a_{2}^{k}\right]\right) \\
\mathcal{F}\left[-\partial_{x_{2}}(-\Delta)^{-1}\left(\operatorname{curl} a^{k}\right)\right] & =i \xi_{2}|\xi|^{-2}\left(-i \xi_{2} \mathcal{F}\left[a_{1}^{k}\right]+i \xi_{1} \mathcal{F}\left[a_{2}^{k}\right]\right) \\
\mathcal{F}\left[\partial_{x_{1}}(-\Delta)^{-1}\left(\operatorname{curl} a^{k}\right)\right] & =-i \xi_{1}|\xi|^{-2}\left(-i \xi_{2} \mathcal{F}\left[a_{1}^{k}\right]+i \xi_{1} \mathcal{F}\left[a_{2}^{k}\right]\right) .
\end{aligned}
$$

Then one observes that

$$
\begin{aligned}
\mathcal{F}\left[a_{1}^{k}\right] & =-i \xi_{1}|\xi|^{-2}\left(i \xi_{1} \mathcal{F}\left[a_{1}^{k}\right]+i \xi_{2} \mathcal{F}\left[a_{2}^{k}\right]\right)+i \xi_{2}|\xi|^{-2}\left(-i \xi_{2} \mathcal{F}\left[a_{1}^{k}\right]+i \xi_{1} \mathcal{F}\left[a_{2}^{k}\right]\right) \\
& =\mathcal{F}\left[\partial_{x_{1}} \alpha^{k}-\partial_{x_{2}} \beta^{k}\right] \\
\mathcal{F}\left[a_{2}^{k}\right] & =-i \xi_{2}|\xi|^{-2}\left(i \xi_{1} \mathcal{F}\left[a_{1}^{k}\right]+i \xi_{2} \mathcal{F}\left[a_{2}^{k}\right]\right)-i \xi_{1}|\xi|^{-2}\left(-i \xi_{2} \mathcal{F}\left[a_{1}^{k}\right]+i \xi_{1} \mathcal{F}\left[a_{2}^{k}\right]\right) \\
& =\mathcal{F}\left[\partial_{x_{2}} \alpha^{k}+\partial_{x_{1}} \beta^{k}\right] .
\end{aligned}
$$

Since $a^{k} \in L^{2}(\mathbb{C})$ we have that $\nabla \alpha^{k}=\nabla\left((-\Delta)^{-1}\left(\operatorname{div} a^{k}\right)\right) \in L^{2}(\mathbb{C}), \nabla^{\perp} \beta^{k}=\nabla^{\perp}\left((-\Delta)^{-1}\left(\operatorname{curl} a^{k}\right)\right) \in$ $L^{2}(\mathbb{C})$ 
Therefore we have

$$
\left\{\begin{array}{l}
\partial_{x_{1}} u-\partial_{x_{1}}\left(\sum_{k=1}^{2} \partial_{x_{k}} \alpha^{k}\right)+\partial_{x_{2}}\left(\sum_{k=1}^{2} \partial_{x_{k}} \beta^{k}\right)=g_{1} \\
\partial_{x_{2}} u-\partial_{x_{2}}\left(\sum_{k=1}^{2} \partial_{x_{k}} \alpha^{k}\right)-\partial_{x_{1}}\left(\sum_{k=1}^{2} \partial_{x_{k}} \beta^{k}\right)=g_{2}
\end{array}\right.
$$

By multiplying first the second equation in (II.21) and summing up the first and second one we get

$$
\left(\partial_{x_{1}}+i \partial_{x_{2}}\right)\left(u-\sum_{k=1}^{2} \partial_{x_{k}} \alpha^{k}-i\left(\sum_{k=1}^{2} \partial_{x_{k}} \beta^{k}\right)\right)=g_{1}+i g_{2}=: g_{\mathbb{C}}
$$

By setting $w_{1}:=\sum_{k=1}^{2} \partial_{x_{k}} \alpha^{k}$ and $w_{2}:=\sum_{k=1}^{2} \partial_{x_{k}} \beta^{k}$ we have

$$
\left\|w_{1}\right\|_{L^{2}(\mathbb{C})}+\left\|w_{2}\right\|_{L^{2}(\mathbb{C})} \lesssim\|f\|_{\dot{W}^{-1,2}} .
$$

and the equation (ㅍ.22) becomes

$$
\partial_{\bar{z}}\left(u+w_{1}+i w_{2}\right)=\frac{g_{\mathbb{C}}}{2}
$$

We set $v:=4 \pi \frac{1}{z} * g$. We have $\partial_{\bar{z}} v=g$ in $\mathcal{S}^{\prime}(\mathbb{C})$. 6 Since $\frac{1}{z} \in L^{2, \infty}$ and $g \in L^{1}$, Young Inequality yields that $v \in L^{2, \infty}$ and

$$
\|v\|_{L^{2, \infty}} \lesssim\left\|\frac{1}{z}\right\|_{L^{2, \infty}}\|g\|_{L^{1}} \lesssim\|g\|_{L^{1}}
$$

The function $h=u+w_{1}+i w_{2}-v$ satisfies $\partial_{\bar{z}} h=0$ in $\mathcal{S}^{\prime}(\mathbb{C})$ and therefore it is holomorphic. This implies that $\Im(h), \Re(h)$ are harmonic functions. By assumption $\Im(h)=w_{2}-\Im(v) \in$ $L^{2, \infty}\left(\mathbb{R}^{2}\right)$ and thus $w_{2}-\Im(v)=0$. Since $\nabla^{\perp}\left(u+w_{1}-\Re v\right)=\nabla\left(w_{2}-\Im(v)\right)$ it follows that there is a constant $c \in \mathbb{R}$ such that $u+w_{1}-\Re v-c=0$. This yields in particular that $u-c \in L^{2, \infty}$. The following estimate holds:

$$
\begin{aligned}
\|u-c\|_{L^{2, \infty}} & =\left\|w_{1}-\Re v\right\|_{L^{2, \infty}} \lesssim\left\|w_{1}\right\|_{L^{2, \infty}}+\|v\|_{L^{2, \infty}} \lesssim\left\|w_{1}\right\|_{L^{2, \infty}}+C\|g\|_{L^{1}} \\
& \leq C\left(\|f\|_{\dot{W}^{-1,2}(\mathbb{C})}+\|g\|_{L^{1}(\mathbb{C})}\right) .
\end{aligned}
$$

We can conclude the proof.

Lemma II.2. Let $g \in L^{1}(\mathbb{C})$ and let $h \in L^{2, \infty}(\mathbb{C})$ satisfy $\partial_{\bar{z}} h=g$ in $\mathcal{S}^{\prime}(\mathbb{C})$. If $\Im h \in L^{2}(\mathbb{C})$, then $\Re h \in L^{2}(\mathbb{C})$ as well and

$$
\|\Re h\|_{L^{2}} \leq C\left(\|g\|_{L^{1}}+\|\Im h\|_{L^{2}}\right) .
$$

\footnotetext{
${ }^{6}$ We recall that $\frac{4}{\pi z}$ satisfies $\partial_{\bar{z}}\left(4 \pi \frac{1}{z}\right)=\delta_{0}$.
} 
Proof of Lemma II.2. Let $\chi \in C_{c}^{\infty}(\mathbb{C})$ such that $\chi=1$ on $B(0,1)$ and $\chi=0$ on $B^{c}(0,2)$. For every $k \geq 1$ we set $\chi_{k}(x)=\chi\left(\frac{x}{k}\right)$. We set $h_{k}=\chi_{k}(x) \varphi_{k} * h$ where $\varphi_{k} \in C_{c}^{\infty}(\mathbb{C})$ is a sequence of mollifiers such that $\int_{\mathbb{R}^{2}} \varphi_{k} d x=1$. We have

$$
\partial_{\bar{z}} h_{k}=\chi_{k} \partial_{\bar{z}}\left(\varphi_{k} * h\right)+\partial_{\bar{z}} \chi_{k} \varphi_{k} * h=: g_{k} .
$$

where

$$
g_{k}=\chi_{k}\left(\partial_{\bar{z}}\left(\varphi_{k} * h\right)+\partial_{\bar{z}} \chi_{k} \varphi_{k} * h\right.
$$

and

$$
\begin{aligned}
\left\|g_{k}\right\|_{L^{1}} & \lesssim\|g\|_{L^{1}}+\frac{1}{k}\left[k^{2}\right]^{1 / 2}\left\|\varphi_{k} * h\right\|_{L^{2, \infty}} \\
& \lesssim\|g\|_{L^{1}}+\left\|\varphi_{k}\right\|_{L^{1}}\|h\|_{L^{2, \infty}} \lesssim\|g\|_{L^{1}}+\|g\|_{L^{1}}\left\|\frac{1}{z}\right\|_{L^{2, \infty}} \\
& \lesssim\|g\|_{L^{1}} .
\end{aligned}
$$

For $\psi \in \mathcal{S}(\mathbb{C})$ we define

$$
\begin{aligned}
\left\langle\frac{1}{(\bar{\xi})^{2}}, \psi(\xi)\right\rangle & :=\int_{\mathbb{C}} \frac{1}{(\bar{\xi})^{2}}\left(\psi(\xi)-\psi(0)-\partial_{\xi_{1}} \psi(0) \xi_{1}-\partial_{\xi_{2}} \psi(0) \xi_{2}\right) d \xi \\
& +\int_{\mathbb{C}} \frac{1}{(\bar{\xi})^{2}} \psi(\xi) d \xi
\end{aligned}
$$

One can see that (II.28) defines a tempered distribution. We set $\xi=\xi_{1}+i \xi_{2}$. Observe that

$$
\mathcal{F}^{-1}\left[\frac{1}{\bar{\xi}^{2}}\right]=\mathcal{F}^{-1}\left[\frac{\xi^{2}}{|\xi|^{4}}\right]=\mathcal{F}^{-1}\left[\frac{\left(\xi_{1}^{2}-\xi_{2}^{2}+2 i \xi_{1} \xi_{2}\right)}{|\xi|^{4}}\right] .
$$

Since $\xi_{1}^{2}-\xi_{2}^{2}+2 i \xi_{1} \xi_{2}$ is homogeneous harmonic polynomial, we can apply Theorem 5 in 3.3 of [20] and deduce the existence of an universal constant $c_{0}$ such that

$$
\mathcal{F}^{-1}\left[\frac{\left(\xi_{1}^{2}-\xi_{2}^{2}+2 i \xi_{1} \xi_{2}\right)}{|\xi|^{4}}\right]=c_{0} \frac{x_{1}^{2}-x_{2}^{2}+2 i x_{1} x_{2}}{|x|^{2}} .
$$

Now we introduce the following tempered distribution

$$
\hat{T}_{k}=-\frac{1}{(\bar{\xi})^{2}} \hat{g}_{k} .
$$

We have

$$
T_{k}=\mathcal{F}^{-1}\left[-\frac{1}{\bar{\xi}^{2}}\right] * g_{k}
$$

It follows then from (1I.30)

$$
\left\|T_{k}\right\|_{L^{\infty}} \lesssim\left\|g_{k}\right\|_{L^{1}}\left\|\frac{x_{1}^{2}-x_{2}^{2}+2 i x_{1} x_{2}}{|x|^{2}}\right\|_{L^{\infty}} \lesssim\|g\|_{L^{1}} .
$$


We also have

$$
\mathcal{F}\left[\partial_{\bar{z}} T_{k}\right]=\frac{i}{\bar{\xi}} \mathcal{F}\left[g_{k}\right]=\mathcal{F}\left[h_{k}\right]
$$

Hence

$$
\begin{aligned}
\left|\Re\left(\int_{\mathbb{C}} h_{k}^{2} d x_{1} d x_{2}\right)\right| & =\left|\Re\left(\int_{\mathbb{C}} h_{k} \partial_{\bar{z}} T_{k} d x_{1} d x_{2}\right)\right| \\
& =\left|-\Re\left(\int_{\mathbb{C}} \partial_{\bar{z}} h_{k} T_{k} d x_{1} d x_{2}\right)\right|=\left|-\Re\left(\int_{\mathbb{C}} g_{k} T_{k} d x_{1} d x_{2}\right)\right| \\
& \leq\left\|g_{k}\right\|_{L^{1}}\left\|T_{k}\right\|_{L^{\infty}} \leq C\|g\|_{L^{1}}^{2}
\end{aligned}
$$

We have

$$
\Re\left(h_{k}^{2}\right)=\left|\Re h_{k}\right|^{2}-\left|\Im h_{k}\right|^{2}
$$

and

$$
\left\|\Im h_{k}\right\|_{L^{2}}^{2}=\int_{\mathbb{C}}\left|\left(\Im h * \varphi_{k}\right) \chi_{k}\right|^{2} \lesssim\|\Im h\|_{L^{2}}^{2} .
$$

From (II.33) and (II.34) we deduce that

$$
\left\|\left(\Re h_{k}\right)\right\|_{L^{2}}^{2} \leq\left\|\Im h_{k}\right\|_{L^{2}}^{2}+\left|\int_{\mathbb{C}} \Re h_{k}^{2} d x\right| \leq\|\Im h\|_{L^{2}}^{2}+C\|g\|_{L^{1}}^{2} .
$$

Up to a subsequence $\Re h_{k}$ converges weakly in $L^{2}$ to $h_{\infty} \in L^{2}\left(\mathbb{R}^{2}\right)$ as $k \rightarrow+\infty$. On the other hand we have $\Re h_{k} \rightarrow \Re h$ in $\mathcal{S}^{\prime}(\mathbb{C})$ and therefore $\Re h=h_{\infty} \in L^{2}(\mathbb{C})$ and by the lower semicontinuity of the $L^{2}$ norm we have

$$
\|(\Re h)\|_{L^{2}}^{2} \leq\|\Im h\|_{L^{2}}^{2}+C\|g\|_{L^{1}}^{2} .
$$

We conclude the proof of Lemma $\llbracket .2$.

By combining Lemmae $\llbracket .1$ and $\llbracket .2$ we can deduce the Brezis-Bourgain Inequality.

Lemma II.3. Let $g \in L^{1}\left(\mathbb{C}, \mathbb{R}^{2}\right)$ and $f \in \dot{W}^{-1,2}\left(\mathbb{C}, \mathbb{R}^{2}\right)$ Let $u \in \mathcal{S}^{\prime}(\mathbb{C}, \mathbb{R})$ be such that

$$
\nabla u=f+g \in\left(\dot{W}^{-1,2}+L^{1}\right)(\mathbb{C}) \quad \text { in } \mathcal{S}^{\prime}(\mathbb{C}) .
$$

Then there is $c \in \mathbb{R}$ such that $u-c \in L^{2}(\mathbb{C})$ and

$$
\|u-c\|_{L^{2}(\mathbb{C})} \leq C\left(\|f\|_{\dot{W}^{-1,2}(\mathbb{C})}+\|g\|_{L^{1}(\mathbb{C})}\right) .
$$




\section{Proof of Lemma II.3.}

From Lemma $\llbracket$ I.1 follows that there is $c \in \mathbb{R}$ such that $u-c \in L^{2, \infty}(\mathbb{C})$ with

$$
\|u-c\|_{L^{2, \infty}(\mathbb{C})} \leq C\left(\|f\|_{\dot{W}^{-1,2}(\mathbb{C})}+\|g\|_{L^{1}(\mathbb{C})}\right) .
$$

Claim 1: $u-c \in L^{2}$ and (II.38) holds.

\section{Proof of Claim 1}

In the proof of Lemma $\llbracket$ we have seen the existence of $\alpha^{k}, \beta^{k} \in \dot{W}^{1,2}(\mathbb{C})(k=1,2)$ such that if we set $w_{1}=\sum_{k=1}^{2} \partial_{x_{k}} \alpha^{k}$ and $w_{2}=\sum_{k=1}^{2} \partial_{x_{k}} \beta^{k}$ we have

$$
\partial_{\bar{z}}\left((u-c)-w_{1}-i w_{2}\right)=\frac{g_{\mathbb{C}}}{2} \quad \text { in } \mathcal{S}^{\prime}(\mathbb{C})
$$

and

$$
\left\|w_{1}\right\|_{L^{2}(\mathbb{C})}+\left\|w_{2}\right\|_{L^{2}(\mathbb{C})} \leq C\|f\|_{\dot{W}^{-1,2}(\mathbb{C})} .
$$

The function $h=(u-c)-w_{1}-i w_{2}$ satisfies the assumptions of Lemma II.2. Therefore we have that $(u-c)-w_{1} \in L^{2}$ with

$$
\int_{\mathbb{C}}\left|u-c-w_{1}\right|^{2} d x \leq \int_{\mathbb{C}}\left|w_{2}\right|^{2} d x+C\|g\|_{L^{1}}^{2}
$$

and

$$
\begin{aligned}
\int_{\mathbb{C}}|u-c|^{2} d x & \lesssim 2\left\|w_{1}\right\|_{L^{2}(\mathbb{C})}^{2}+2\left\|w_{2}\right\|_{L^{2}(\mathbb{C})}^{2}+C\|g\|_{L^{1}}^{2} \\
& \leq C\left(\|f\|_{\dot{W}^{-1,2}(\mathbb{C})}^{2}+\|g\|_{L^{1}}^{2}\right) .
\end{aligned}
$$

We conclude the proof.

Remark II.5. We observe that if in the Lemma II.3 $\nabla u=\nabla^{\perp} v+g$ with $g \in L^{1}$ and $v \in L^{2}$ then we simply get the estimate

$$
\int_{\mathbb{C}}|u-c|^{2} d x \leq\|v\|_{L^{2}(\mathbb{C})}^{2}+C\|g\|_{L^{1}}^{2}
$$

namely the constant in front of $\|v\|_{L^{2}(\mathbb{C})}^{2}$ is 1 .

\section{Regularity of solutions to $\operatorname{div}(S \nabla u)=0:$ Proof of theorem I.1.}

In this section we are going to investigate the regularity of $L^{2}$ solutions to the following system

$$
\operatorname{div}(S \nabla u)=0 \text { in } \mathcal{D}^{\prime}(\mathbb{C})
$$


where $S \in \dot{W}^{1,2}(\mathbb{C}, O(n))$ with $S^{2}=I d$.

It has been shown in [12] that there exists solutions $u \in W_{l o c}^{1,1}(B(0,1))$ of $\operatorname{div}(A \nabla u)=0$ in $\mathcal{D}^{\prime}(B(0,1))$ where $A$ is a uniformly elliptic and continuous matrix which is in none of the spaces $W_{l o c}^{1, p}(B(0,1))$ for any $p>1$.

Actually they construct a counter-example of a matrix $A$ which turns out to be also in $W^{1,2}(B(0,1))$. The matrix $A(x)=\left(a_{i j}(x)\right)_{\substack{1 \leq i \leq n \\ 1 \leq j \leq n}}$ is defined as follows

$$
a_{i j}(x)=\delta_{i j}+\alpha(|x|)\left(\delta_{i j}-\frac{x_{i} x_{j}}{|x|^{2}}\right)
$$

where

$$
\alpha(r)=\frac{-\beta n}{(n-1)\left(\log \frac{r_{0}}{r}\right)}+\frac{\beta(\beta+1)}{(n-1)\left(\log \frac{r_{0}}{r}\right)^{2}} .
$$

where $r_{0}$ is large enough so that $\alpha \geq-\frac{1}{2}$ and $\beta>1$.

Clearly $a_{i j} \in L^{2}(B(0,1))$. A direct computation for any $i, j, k$ gives

$$
\frac{\partial a_{i j}}{\partial x_{k}}=\alpha^{\prime}(|x|) \frac{x_{k}}{|x|}\left(\delta_{i j}-\frac{x_{i} x_{j}}{|x|^{2}}\right)-\alpha(|x|)\left(\frac{\left(\delta_{i k} x_{j}+\delta_{j k} x_{i}\right)|x|^{2}-2 x_{k} x_{i} x_{j}}{|x|^{4}}\right) .
$$

Therefore

$$
\left|\frac{\partial a_{i j}}{\partial x_{k}}(x)\right| \leq C \frac{1}{r} \frac{1}{\log \left(\frac{r_{0}}{r}\right)} .
$$

Since $\frac{1}{r} \frac{1}{\log \left(\frac{r_{0}}{r}\right)} \in L^{2}(B(0,1))$ then $\nabla a_{i j} \in L^{2}(B(0,1))$ as well. It is proved in 12] that

$$
u(x)=x_{1} \frac{1}{r^{2} \log \left(\frac{r_{0}}{r}\right)^{\beta}} \in L^{2}(B(0,1)) \quad \text { solves } \quad \sum_{i j=1}^{2} \partial_{x_{i}}\left(a_{i j} \partial_{x_{j}} u\right)=0 .
$$

The function $u$ defined in (III.46) is not in the spaces $W_{l o c}^{1, p}(B(0,1))$ for any $p>1$.

We are now proving the following result

Theorem III.6. There is an $\varepsilon_{0}>0$ such that if $S \in \dot{W}^{1,2}(\mathbb{C}, O(n))$ with $S^{2}=I_{n}$ and $\|\nabla S\|_{L^{2}(\mathbb{C})} \leq \varepsilon_{0}$ then there is $Q \in \dot{W}^{1,2}(\mathbb{C}, S O(n))$ such that

$$
S=Q S^{0} Q^{-1}
$$

where

$$
S^{0}=\left(\begin{array}{c|c}
I_{m \times m} & 0_{m \times n-m} \\
\hline 0_{n-m \times m} & -I_{n-m \times n-m}
\end{array}\right)
$$

with $m \leq n$ and

$$
\|\nabla Q\|_{L^{2}} \leq C\|\nabla S\|_{L^{2}}
$$

where $C>0$ only depends on $n$. 
Proof of Theorem III.6. Let $S \in \dot{W}^{1,2}\left(\mathbb{R}^{2}, O(n)\right)$ be with $S^{2}=I_{n}$.

We have $\operatorname{det} S, \operatorname{Trace}(S) \in \dot{W}^{1,2}\left(\mathbb{R}^{2}, \mathbb{Z}\right)$. Precisely

$$
\operatorname{det} S=(-1)^{n-m}, \text { and } \operatorname{Trace}(S)=2 m-n
$$

where $m=\#$ positive eigenvalues and $n-m=\#$ negative eigenvalues (we recall that the eigenvalues of $S$ can be either 1 or -1$)$. Since $\operatorname{det} S$, Trace $(S) \in \dot{W}^{1,2}\left(\mathbb{R}^{2}\right)$ it follows that det $S$ and $\operatorname{Trace}(S)$ are both constant a.e. in $\mathbb{R}^{2}$.

We set

$$
P_{R}:=\frac{I-S}{2} \quad \text { and } \quad P_{L}=\frac{I+S}{2}
$$

$P_{L}, P_{R}$ are idempotent since $(I-S)^{2}=S^{2}-2 S+I=2(I-S)$ and $(I+S)^{2}=2(I+S)$ and the ranks of $P_{L}$ and $P_{R}$ are constant.

We can see $P_{L}$ (resp. $\left.P_{R}\right)$ as $\dot{W}^{1,2}$ maps with values into the Grassmanian $G r_{m}\left(\mathbb{R}^{n}\right)$ (resp. $\left.G r_{n-m}\left(\mathbb{R}^{n}\right)\right)$ of nonoriented $m$-planes (resp. $n-m$-planes) in $\mathbb{R}^{n}$.

By applying Lemma 5.1.4 in Hélein book [10 there is an $\varepsilon_{0}>0$ such that one can find two $\dot{W}^{1,2}\left(\mathbb{R}^{2}\right)$ orthonormal basis $e=e_{1}, \ldots, e_{m}$ and $f_{1}, \ldots, f_{n-m}$ of $\operatorname{Im}\left(P_{L}\right)$ and $\operatorname{Im}\left(P_{R}\right)$ respectively such that

$$
\left\|\nabla e_{i}\right\|_{L^{2}} \leq C\left\|\nabla P_{L}\right\|_{L^{2}} \quad \text { and } \quad\left\|\nabla f_{j}\right\|_{L^{2}} \leq C\left\|\nabla P_{R}\right\|_{L^{2}}
$$

for $i=1, \ldots, m$ and $j=1, \ldots, n-m$.

Let $\left(\epsilon_{k}\right)_{k=1, \ldots, n}$ be the canonical basis of $\mathbb{R}^{n}$. Let $Q_{L} \in \dot{W}^{1,2}\left(\mathbb{R}^{2}, \mathcal{M}_{n \times n}\right)$ and $Q_{R} \in$ $\dot{W}^{1,2}\left(\mathbb{R}^{2}, \mathcal{M}_{n \times n}\right)$ be defined by

$$
Q_{L}=\left(\begin{array}{c|c}
Q^{L} & 0_{n \times n-m} \\
\hline 0_{n-m \times m} & 0
\end{array}\right)
$$

and

$$
Q_{R}=\left(\begin{array}{c|c}
0 & 0_{n \times n-m} \\
\hline 0_{n-m \times m} & Q^{R}
\end{array}\right)
$$

with $Q^{L} \in S O(m), Q^{R} \in S O(n-m)$ and

$$
\left\{Q_{L} \epsilon_{k}, \quad k=1, \ldots, m\right\}=\left\{e_{j}, \quad j=1, \ldots m\right\}
$$

and

$$
\left\{Q_{R} \epsilon_{k}, \quad k=m+n, \ldots, n\right\}=\left\{e_{j}, \quad j=1, \ldots n-m\right\}
$$

Moreover

$$
P^{L}=Q_{L}^{-1} P_{L} Q_{L} \quad \text { and } \quad P^{R}=Q_{R}^{-1} P_{R} Q_{R}
$$

where

$$
P^{L}=\left(\begin{array}{c|c}
I_{m \times m} & 0_{m \times n-m} \\
\hline 0_{n-m \times m} & 0_{n-m \times n-m}
\end{array}\right)
$$


and

$$
P^{R}=\left(\begin{array}{c|c}
0_{m \times m} & 0_{m \times n-m} \\
\hline 0_{n-m \times m} & I_{n-m \times n-m}
\end{array}\right)
$$

We define

$$
Q=\left(\begin{array}{c|c}
Q_{L} & 0_{n \times n-m} \\
\hline 0_{n-m \times m} & Q_{R}
\end{array}\right) .
$$

By construction we have $Q^{t} Q=I d, S^{0}=Q^{-1} S Q$ and

$$
\|\nabla Q\|_{L^{2}} \leq C\|\nabla S\|_{L^{2}} \text {. }
$$

This concludes the proof of Theorem \II.6.

Next we show how theorem I.1 implies theorem [.3. More precisely we establish that (III.43) is equivalent to (I.7) for a suitable choice of $f$.

Proposition III.1. Let $S \in W^{1,2}(\mathbb{C}, O(n))$ with $S^{2}=I_{n}$ and let $u \in L^{2}\left(\mathbb{C}, \mathbb{R}^{n}\right)$ be a solution of

$$
\operatorname{div}(S \nabla u)=0 \text { in } \mathcal{D}^{\prime}(\mathbb{C})
$$

Then there exists $v \in L^{2}\left(\mathbb{C}, \mathbb{R}^{n}\right)$ such that $\nabla^{\perp} v=S \nabla u$ in $\mathcal{D}^{\prime}(\mathbb{C})$. Moreover the function $f=u+i v$ satisfies

$$
\left\{\begin{array}{c}
P_{L} \frac{\partial f}{\partial z}=0 \text { in } \mathcal{D}^{\prime}(\mathbb{C}) \\
P_{R} \frac{\partial}{\partial \bar{z}} f=0 \text { in } \mathcal{D}^{\prime}(\mathbb{C}) .
\end{array},\right.
$$

where $P_{L}, P_{R}$ are given by (III.50).

Proof of Proposition III.1. Let $v \in \mathcal{D}^{\prime}(\mathbb{C})$ be such that $\nabla^{\perp} v=S \nabla u$ in $\mathcal{D}^{\prime}(\mathbb{C})$. It holds $\nabla^{\perp} v=\nabla(S u)-\nabla S u \in \dot{W}^{-1,2}+L^{1}$. Lemma $\llbracket .3$ gives that $v \in L^{2}(\mathbb{C})$. We have

$$
\left\{\begin{array}{l}
S \partial_{x_{1}} u=-\partial_{x_{2}} v \\
S \partial_{x_{2}} u=\partial_{x_{1}} v
\end{array}\right.
$$

Therefore

$$
S \partial_{x_{1}}(u+i v)=i \partial_{x_{2}}(u+i v) .
$$

Let us introduce $f: \mathbb{C} \rightarrow \mathbb{C}^{n}$ given by $f=u+i v$. Obviously $f$ satisfies:

$$
\left\{\begin{array}{c}
S \partial_{x_{1}} f-i \partial_{x_{2}} f=0 \\
S i \partial_{x_{2}} f-\partial_{x_{1}} f=0 .
\end{array}\right.
$$

By first subtracting and then summing the two equations in (III.62) we deduce that

$$
(S+I) \partial_{z} f=0, \quad(S-I) \partial_{\bar{z}} f=0
$$

Therefore $f$ satisfies (III.59) and we conclude the proof. 


\section{III.1 Proof of theorem I.1: the case $n=2$}

In this section we focus our attention to the case where the function $u$ takes values in $\mathbb{R}^{2}$, since as we will see the formulation will become simpler and maybe more enlightening .

Let $Q \in \dot{W}^{1,2}(\mathbb{C}, S O(2))$ then a classical result by Carbou gives the existence of $\alpha \in \dot{W}^{1,2}(\mathbb{C}, \mathbb{R})$ such that

$$
Q(x)=\left(\begin{array}{cc}
\cos (\alpha(x)) & -\sin (\alpha(x)) \\
\sin (\alpha(x)) & \cos (\alpha(x))
\end{array}\right) .
$$

We also set

$$
S^{0}=\left(\begin{array}{cc}
1 & 0 \\
0 & -1
\end{array}\right)
$$

Next we re-formulate the system (I.6) in the $n=2$ case. Precisely we have

Proposition III.2. Let $S \in \dot{W}^{1,2}(\mathbb{C}, O(2))$ with $S^{2}=I d$ and $\|\nabla S\|_{L^{2}(\mathbb{C})} \leq \varepsilon_{0}$ (with $\varepsilon_{0}>0$ as in Theorem III.6). Let $Q \in S O(2)$ as in (III.64) such that $S=Q^{-1} S^{0} Q$ and let $u, v$ be as in the statement of Proposition III.1. Then function $f: \mathbb{C} \rightarrow\left(\mathbb{C}^{2}\right)$

$$
f:=S^{0} Q u+i Q v
$$

satisfies the following equation

$$
\partial_{z} f=\left(\begin{array}{cc}
0 & 1 \\
-1 & 0
\end{array}\right) \partial_{z} \alpha \bar{f}
$$

Proof of proposition III.2 Let $u \in L^{2}\left(\mathbb{R}^{2}\right)$ be a solution of (III.58) and $v \in L^{2}(\mathbb{C})$ be such that

$$
\nabla^{\perp} v=S \nabla u
$$

Since $S=Q^{-1} S^{0} Q$ we can write $(\underline{\text { III.68) }}$ ) as

$$
Q \nabla^{\perp} v=S^{0} Q \nabla u
$$

We set $f_{\Re}:=S^{0} Q u$ and $f_{\Im}:=Q v$. From the fact that $S^{0} Q \nabla u-Q \nabla^{\perp} v=0$ it follows

$$
\nabla\left(f_{\Re}\right)-\nabla^{\perp}\left(f_{\Im}\right)=\nabla\left(S^{0} Q\right) u-\nabla^{\perp} Q v=S^{0} \nabla Q Q^{-1} S^{0} f_{\Re}-\nabla^{\perp} Q Q^{-1} f_{\Im} .
$$

Therefore

$$
\left\{\begin{array}{l}
\partial_{x_{1}} f_{\Re}+\partial_{x_{2}} f_{\Im}=S^{0}\left(\partial_{x_{1}} Q\right) Q^{-1} S^{0} f_{\Re}+\partial_{x_{2}} Q Q^{-1} f_{\Im} \\
\partial_{x_{2}} f_{\Re}-\partial_{x_{1}} f_{\Im}=S^{0}\left(\partial_{x_{2}} Q\right) Q^{-1} S^{0} f_{\Re}-\partial_{x_{1}} Q Q^{-1} f_{\Im}
\end{array}\right.
$$


We have

$$
\begin{aligned}
\nabla Q Q^{-1} & =\left(\begin{array}{cc}
0 & -1 \\
1 & 0
\end{array}\right) \nabla \alpha \\
S^{0} \nabla Q Q^{-1} S^{0} & =\left(\begin{array}{cc}
0 & 1 \\
-1 & 0
\end{array}\right) \nabla \alpha .
\end{aligned}
$$

We have

$$
\left\{\begin{array}{c}
\partial_{x_{1}} f_{\Re}+\partial_{x_{2}} f_{\Im}=\left(\begin{array}{cc}
0 & 1 \\
-1 & 0
\end{array}\right) \partial_{x_{1}} \alpha f_{\Re}+\left(\begin{array}{cc}
0 & -1 \\
1 & 0
\end{array}\right) \partial_{x_{2}} \alpha f_{\Im} \\
-\partial_{x_{2}} f_{\Re}+\partial_{x_{1}} f_{\Im}=\left(\begin{array}{cc}
0 & -1 \\
1 & 0
\end{array}\right) \partial_{x_{2}} \alpha f_{\Re}+\left(\begin{array}{cc}
0 & -1 \\
1 & 0
\end{array}\right) \partial_{x_{1}} \alpha f_{\Im} .
\end{array}\right.
$$

From (III.71) it follows

$$
\partial_{x_{1}} f_{\Re}+\partial_{x_{2}} f_{\Im}-i\left(\partial_{x_{2}} f_{\Re}-\partial_{x_{1}} f_{\Im}\right)=\left(\begin{array}{cc}
0 & 1 \\
-1 & 0
\end{array}\right)\left(\partial_{x_{1}} \alpha-i \partial_{x_{2}} \alpha\right)\left(f_{\Re}-i f_{\Im}\right) .
$$

Hence

$$
\partial_{z} f=\left(\begin{array}{cc}
0 & 1 \\
-1 & 0
\end{array}\right) \partial_{z} \alpha \bar{f}
$$

This concludes the proof of proposition III.2.

Now we present the regularity of the equation (III.67) and therefore of (III.58). We would like first to explain the reasons why the equation (III.72) does not fall within the classical theory of systems with a $L^{2}$ potential.

Let us represent a function $f=u+i v$ with $u=\left(u_{1}, u_{2}\right), v=\left(v_{1}, v_{2}\right)$ as

$$
f=\left(\begin{array}{l}
u_{1}+i v_{1} \\
u_{2}+i v_{2}
\end{array}\right) .
$$

We observe that the equation (III.67) can be written as

$$
\left\{\begin{array}{l}
\partial_{z}\left(u_{1}+i v_{1}\right)=\partial_{z} \alpha\left(u_{2}-i v_{2}\right) \\
\partial_{z}\left(u_{2}+i v_{2}\right)=-\partial_{z} \alpha\left(u_{1}-i v_{1}\right) .
\end{array}\right.
$$

The system (III.73) is of the form

$$
\left\{\begin{array}{l}
\partial_{z} \phi=\omega \bar{\psi} \\
\partial_{z} \psi=-\omega \bar{\phi}
\end{array}\right.
$$


where $\omega=\partial_{z} \alpha \in L^{2}(\mathbb{C}, \mathbb{C})$. The difficulty is that in the right hand side of (III.74) there are the conjugate of the unknows $(\phi, \psi)$. Suppose we would have instead a system of the form

$$
\left\{\begin{array}{c}
\partial_{z} \phi=\partial_{z} \alpha \psi \\
\partial_{z} \psi=-\partial_{z} \alpha \phi .
\end{array}\right.
$$

Then the function $\Phi:=\left(\phi_{1}, \phi_{2}\right)$ solves

$$
\partial_{z} \Phi=\Omega \Phi
$$

where

$$
\Omega=\left(\begin{array}{cc}
0 & \partial_{z} \alpha \\
-\partial_{z} \alpha & 0
\end{array}\right)=\partial_{z} Q Q^{-1}
$$

Hence we would deduce $\partial_{z}(Q \Phi)=0$ which would imply that $\Phi \in W_{l o c}^{1,2}$. Unfortunately the multiplication of $\Phi$ solving (III.74) by a matrix in $S O(2)$ does not permit to absorb the potential $\Omega$ which is the case of interest in the present work. Therefore we have to find a different Lie group that permits us to absorb the potential.

To this purpose we introduce the algebra of Quaternions. We recall standard notations regarding this algebra that we denote by $\mathbb{H}$ :

$$
\mathbb{H}:=\left\{a+b i+c j+d k, \quad(a, b, c, d) \in \mathbb{R}^{4}\right\},
$$

where $i, j$ and $k$ are the fundamental quaternion units satisfying $i^{2}=j^{2}=k^{2}=-1$ and $i j=-j i=k, j k=-k j=i$ and $k i=-i k=j$. The set $\mathbb{H}$ of all quaternions is a vector space over the real numbers with dimension 4 . The conjugate of $\mathfrak{q} \in \mathbb{H}$ is the quaternion $q^{*}=a-b i-c j-d k$. The reciprocal of $\mathfrak{q} \in \mathbb{H}^{*}$ is $q^{-1}=\frac{\mathfrak{q}^{*}}{|\mathfrak{q}|}$, where $|\mathfrak{q}|=\sqrt{\mathfrak{q} \mathfrak{q}^{*}}$ is the norm of $\mathfrak{q}$.

Given $\mathfrak{q} \in \mathbb{H}, \mathfrak{q}=q_{1}+q_{2} i+q_{3} j+q_{4} k$ we set

$$
\Pi_{i}(\mathfrak{q})=q_{2} i \text { and } \Pi_{j k}(\mathfrak{q})=q_{3} j+q_{4} k .
$$

We also denote by $\mathbb{H}_{p}$ the quaternion of the form $\mathfrak{q}=q_{2} i+q_{3} j+q_{4} k$ (the pure quaternions) and $\mathcal{U}(\mathbb{H}):=\{\mathfrak{q} \in \mathbb{H}: \quad|\mathfrak{q}|=1\} . \mathbb{H}_{p}$ is the Lie Algebra of the Lie Group $\mathcal{U}(\mathbb{H})$.

Finally given $\mathfrak{f}: \mathbb{C} \rightarrow \mathbb{H}$ we introduce the following differential operators (CauchyRiemann-Fueter operators):

$$
\begin{aligned}
\partial_{L} \mathfrak{f} & :=2^{-1}\left(\partial_{x_{1}} \mathfrak{f}-i \partial_{x_{2}} \mathfrak{f}\right) \\
\partial_{R} f & :=2^{-1}\left(\partial_{x_{1}} \mathfrak{f}-\partial_{x_{2}} \mathfrak{f} i\right) .
\end{aligned}
$$

and

$$
\begin{aligned}
\bar{\partial}_{L} \mathfrak{f} & :=2^{-1}\left(\partial_{x_{1}} \mathfrak{f}+i \partial_{x_{2}} \mathfrak{f}\right) \\
\bar{\partial}_{R} f & :=2^{-1}\left(\partial_{x_{1}} \mathfrak{f}+\partial_{x_{2}} \mathfrak{f} i\right) .
\end{aligned}
$$


We observe that if $\mathfrak{f}$ takes values in $\mathbb{C}$ then

$$
\partial_{L} \mathfrak{f}=\partial_{R} \mathfrak{f}=\partial_{z} \mathfrak{f} \text { and } \bar{\partial}_{L} \mathfrak{f}=\bar{\partial}_{R} \mathfrak{f}=\partial_{\bar{z}} \mathfrak{f} .
$$

We are going to rewrite the equation (III.67) and therefore (III.73) using the quaternion valued functions.

Lemma III.4. Let

$$
f=\left(\begin{array}{c}
u_{1}+i v_{1} \\
u_{2}+i v_{2}
\end{array}\right)
$$

be a solution of (ㅍ․67) then the quaternion

$$
\mathfrak{f}=u_{1}+v_{1} i+u_{2} j+v_{2} k
$$

satisfies

$$
\partial_{L} \mathfrak{f}=-\partial_{z} \alpha j \mathfrak{f}
$$

Proof of lemma III.4. We have seen that the equation (III.67) is equivalent to the system (III.73). Such a system can also be written using the $\partial_{L}$ operator, which coincides with $\partial_{R}$ at this stage since the variables $u_{1}+i u_{2}$ and $v_{1}+i v_{2}$ are $\mathbb{C}$-valued.

$$
\left\{\begin{array}{l}
\partial_{L}\left(u_{1}+i v_{1}\right)=\partial_{z} \alpha\left(u_{2}-i v_{2}\right) \\
\partial_{L}\left(u_{2}+i v_{2}\right)=-\partial_{z} \alpha\left(u_{1}-i v_{1}\right) .
\end{array}\right.
$$

We multiply from the right the second equation in (III.82) by $j$ and we get (recall that $i j=k=-j i)$

$$
\partial_{L}\left(u_{2} j+v_{2} k\right)=-\partial_{z} \alpha j\left(u_{1}+i v_{1}\right) .
$$

On another hand we can write the first equation in (III.82) as follows:

$$
\partial_{L}\left(u_{1}+i v_{1}\right)=-\partial_{z} \alpha j^{2}\left(u_{2}-i v_{2}\right)=-\partial_{z} \alpha j\left(u_{2} j+v_{2} k\right) .
$$

By summing (III.83) and (III.84) we find

$$
\partial_{L}\left(u_{1}+v_{1} i+u_{2} j+v_{2} k\right)=-\partial_{z} \alpha j\left(u_{1}+v_{1} i+u_{2} j+v_{2} k\right) .
$$

Hence we get (III.81) and we can conclude. 


\section{III.2 Bootstrap test for $\partial_{L} \mathfrak{f}=\partial_{z} \alpha j \mathfrak{f}$}

In the sequel up to exchange $\alpha$ and $-\alpha$ we study the following equation

$$
\partial_{L} \mathfrak{f}=\partial_{z} \alpha j \mathfrak{f}
$$

Actually all that is proved in this section also holds for a system of the form

$$
\partial_{L} \mathfrak{f}=\Omega j \mathfrak{f}
$$

where $\Omega \in L^{2}(\mathbb{C}, \mathbb{C})$.

The first main goal of this section is to show that the operator

$$
\mathfrak{f} \in L^{2}(\mathbb{C}, \mathbb{H}) \mapsto \partial_{L} \mathfrak{f}-\partial_{z} \alpha j \mathfrak{f}
$$

is injective if the $L^{2}$ norm of $\partial_{z} \alpha$ is sufficiently small. This is what we call the "bootstrap test".

Theorem III.7. There exists $\varepsilon_{0}>0$ such that for every $\alpha \in \dot{W}^{1,2}(\mathbb{C}, \mathbb{R})$ satisfying $\|\nabla \alpha\|_{L^{2}} \leq \varepsilon_{0}$ and every $\mathfrak{f} \in L^{2}(\mathbb{C}, \mathbb{H})$ solving

$$
\partial_{L} \mathfrak{f}=\partial_{z} \alpha j \mathfrak{f}
$$

then $\mathfrak{f} \equiv 0$.

Before going to the proof of theorem \II.7 we will introduce a nonlinear operator $\mathbf{N}$.

Let $\mathfrak{q} \in \mathcal{U}(\mathbb{H})$. We multiply the equation (III.88) ) on the left by $\mathfrak{q}$ :

$$
\mathfrak{q}\left[\partial_{x_{1}} \mathfrak{f}-i \partial_{x_{2}} \mathfrak{f}\right]=\mathfrak{q}\left[\partial_{x_{1}} \alpha-\partial_{x_{2}} \alpha i\right] j \mathfrak{f} .
$$

Observe that

$$
\mathfrak{q}\left[\partial_{x_{1}} \mathfrak{f}-i \partial_{x_{2}} \mathfrak{f}\right]=\partial_{x_{1}}[\mathfrak{q} \mathfrak{f}]-\partial_{x_{2}}[\mathfrak{q} i \mathfrak{f}]-\partial_{x_{1}} \mathfrak{q} \mathfrak{f}+\partial_{x_{2}} \mathfrak{q} i \mathfrak{f}
$$

By combining (III.89) and (III.90) we get

$$
\begin{aligned}
\partial_{x_{1}}[\mathfrak{q} \mathfrak{f}]-\partial_{x_{2}}[\mathfrak{q} i \mathfrak{f}] & =\mathfrak{q}\left[\partial_{x_{1}} \alpha-\partial_{x_{2}} \alpha i\right] j \mathfrak{f} \\
& +\partial_{x_{1}} \mathfrak{q} \mathfrak{f}-\partial_{x_{2}} \mathfrak{q} i \mathfrak{f} \\
& =\mathfrak{q}\left[\partial_{x_{1}} \alpha j-\partial_{x_{2}} \alpha k+\mathfrak{q}^{-1} \partial_{x_{1}} \mathfrak{q}-\mathfrak{q}^{-1} \partial_{x_{2}} \mathfrak{q} i\right] \mathfrak{f} .
\end{aligned}
$$

We observe that since $|\mathfrak{q}|=1$ then $\mathfrak{q}^{-1} \partial_{x_{i}} \mathfrak{q} \in \mathbb{H}_{p}$.

We introduce the following operator

$$
\begin{aligned}
\mathbf{N}: & \dot{W}^{1,2}(\mathbb{C}, \mathcal{U}(\mathbb{H})) \rightarrow \dot{W}^{-1,2}(\mathbb{C}, \operatorname{Span}\{i\}) \times L^{2}(\mathbb{C}, \operatorname{Span}\{j, k\}) \\
& \mathfrak{q} \mapsto\left(\Pi_{i}\left(\partial_{x_{1}}\left(\mathfrak{q}^{-1} \partial_{x_{1}} \mathfrak{q}\right)+\partial_{x_{2}}\left(\mathfrak{q}^{-1} \partial_{x_{2}} \mathfrak{q}\right)\right), \Pi_{j k}\left(\mathfrak{q}^{-1} \partial_{x_{1}} \mathfrak{q}-\mathfrak{q}^{-1} \partial_{x_{2}} \mathfrak{q} i\right)\right.
\end{aligned}
$$

We shall prove the following result. 
Lemma III.5. There is $\varepsilon_{0}>0$ and $C>0$ such that for any choice of $\omega \in \dot{W}^{-1,2}(\mathbb{C}, i \mathbb{R})$ and $\mathfrak{g} \in L^{2}(\mathbb{C}, \operatorname{Span}\{j, k\})$ satisfying

$$
\|\omega\|_{\dot{W}^{-1,2}} \leq \varepsilon_{0}, \quad\|\mathfrak{g}\|_{L^{2}} \leq \varepsilon_{0}
$$

then there is $\mathfrak{q} \in \dot{W}^{1,2}(\mathbb{C}, \mathcal{U}(\mathbb{H}))$.

$$
\mathbf{N}(\mathfrak{q})=(\omega, \mathfrak{g})
$$

and

$$
\|\nabla \mathfrak{q}\|_{L^{2}} \leq C\left(\|\omega\|_{\dot{W}^{-1,2}}+\|\mathfrak{g}\|_{L^{2}}\right) .
$$

In order to prove lemma III.5 we shall need to introduce some notations and establish some intermediate results.

As in [5, Proof of Theorem 1.2, Step 4], by an approximation argument it suffices to prove Lemma $\amalg .5$ assuming that $\omega$ and $\mathfrak{g}$ are slightly more regular.

We fix $2<p<+\infty$ and for $\varepsilon>0$ we introduce

$$
\mathcal{U}_{\varepsilon}:=\left\{\begin{array}{c}
(\omega, \mathfrak{g}) \in \dot{W}^{-1, p} \cap \dot{W}^{-1, p^{\prime}}(\mathbb{C}, i \mathbb{R}) \times L^{p} \cap L^{p^{\prime}}(\mathbb{C}, \operatorname{Span}\{j, k\}) \\
\|\omega\|_{\dot{W}^{-1,2}}+\|\mathfrak{g}\|_{L^{2}} \leq \varepsilon
\end{array}\right\}
$$

where $\frac{1}{p}+\frac{1}{p^{\prime}}=1$.

For constants $\varepsilon, \Theta>0$ let $\mathcal{V}_{\varepsilon, \Theta} \subseteq \mathcal{U}_{\varepsilon}$ be the set where we have the decomposition (III.94) with the estimates

$$
\begin{aligned}
\|\nabla \mathfrak{q}\|_{2} & \leq \Theta\left(\|\omega\|_{\dot{W}^{-1,2}}+\|\mathfrak{g}\|_{L^{2}}\right) \\
\|\nabla \mathfrak{q}\|_{p} & \leq \Theta\left(\|\omega\|_{\dot{W}^{-1, p}}+\|\mathfrak{g}\|_{L^{p}}\right), \\
\|\nabla \mathfrak{q}\|_{p^{\prime}} & \leq \Theta\left(\|\omega\|_{\dot{W}^{-1, p^{\prime}}}+\|\mathfrak{g}\|_{L^{p^{\prime}}}\right) .
\end{aligned}
$$

That is

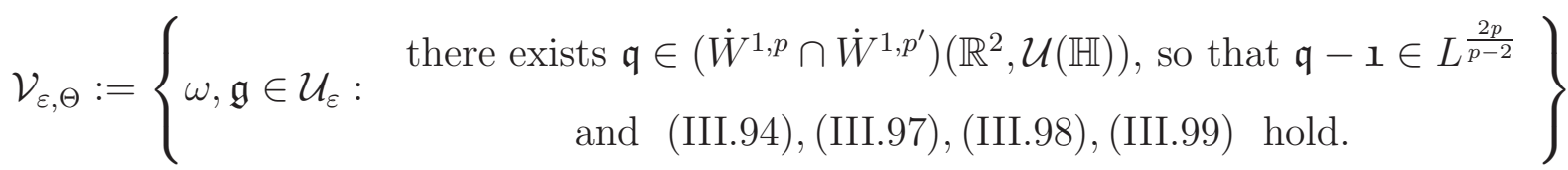

7 The strategy to prove lemma \II.5 follows the one K. Uhlenbeck introduced in 21] to construct Coulomb gauges in critical dimensions. Precisely lemma III.5 is going to be a consequence of the following proposition.

Proposition III.3. There exist $\Theta>0$ and $\varepsilon>0$ so that $\mathcal{V}_{\varepsilon, \Theta}=\mathcal{U}_{\varepsilon}$.

\footnotetext{
${ }^{7}$ Note that (III.97) could actually be deduced from (III.98), (III.99) by interpolation.
} 
Proof of Proposition III.3. Proposition III.3 follows, once we show the following four properties

(i) $\mathcal{U}_{\varepsilon}$ is connected.

(ii) $\mathcal{V}_{\varepsilon, \Theta}$ is nonempty.

(iii) For any $\varepsilon, \Theta>0, \mathcal{V}_{\varepsilon, \Theta}$ is a relatively closed subset of $\mathcal{U}_{\varepsilon}$.

(iv) There exist $\Theta>0$ and $\varepsilon>0$ so that $\mathcal{V}_{\varepsilon, \Theta}$ is a relatively open subset of $\mathcal{U}_{\varepsilon}$.

Property (i) is clear, since $\mathcal{U}_{\varepsilon}$ is obviously starshaped with center 0.

Property (ii) is also obvious since : $(0,0) \in \mathcal{V}_{\varepsilon, \Theta}$.

The closedness property (iii) follows almost verbatim as in [5, Proof of Theorem 1.2, Step 1, p.1315]: there one replaces $(-\Delta)^{1 / 4}$ by $\nabla$. Observe that a uniform bound of the $L^{p}$-norm as in (III.99) implies by Sobolev embedding in particular a uniform bound of $\mathfrak{q}-1$ in $L^{\frac{2 p}{p-2}}\left(\mathbb{R}^{2}\right)$.

We show now the openness property (iv). For this let $\omega_{0}, \mathfrak{g}_{0}$ be arbitrary in $\mathcal{V}_{\varepsilon, \Theta}$, for some $\varepsilon, \Theta>0$ chosen below.

Let $\mathfrak{q}_{0} \in \dot{W}^{1, p} \cap \dot{W}^{1, p^{\prime}}(\mathbb{C}, \mathcal{U}(\mathbb{H})), \mathfrak{q}_{0}-1 \in L^{\frac{2 p}{p-2}}(\mathbb{C})$ so that the decomposition (II.94) as well as the estimates (III.97), (III.98) and (II.99) are satisfied for $\omega_{0}$ and $\mathfrak{g}_{0}$.

We consider perturbations of $\mathfrak{q}_{0}$ of the form $\mathfrak{q}=\mathfrak{q}_{0} e^{\mathfrak{u}}$ where $\mathfrak{u} \in\left(\dot{W}^{1, p} \cap \dot{W}^{1, p^{\prime}} \cap\right.$ $\left.L^{\frac{2 p}{p-2}}\right)\left(\mathbb{C}, \mathbb{H}_{p}\right)$. Observe that the exponent $p>2$ has been chosen in particular to ensure $\mathfrak{u} \in\left(C^{0} \cap L^{\infty}\right)(\mathbb{C})$ with uniform estimates and $\mathfrak{q}_{0} e^{\mathfrak{u}}-\mathfrak{1} \in L^{\frac{2 p}{p-2}}$. 
8

We set

$$
\begin{aligned}
\tilde{N}_{\mathfrak{q}_{0}}:\left(\dot{W}^{1, p} \cap \dot{W}^{1, p^{\prime}} \cap L^{\frac{2 p}{p-2}}\right)\left(\mathbb{C}, \mathbb{H}_{p}\right) & \rightarrow\left(i\left(\dot{W}^{-1, p} \cap \dot{W}^{-1, p^{\prime}}\right)(\mathbb{C}), L^{p} \cap L^{p^{\prime}}(\mathbb{C}, \operatorname{span}\{j, k\})\right) \\
\mathfrak{u} & \mapsto \tilde{N}_{\mathfrak{q}_{0}}(\mathfrak{u}):=\mathbf{N}\left(\mathfrak{q}_{0} e^{\mathfrak{u}}\right) .
\end{aligned}
$$

We will write $\mathfrak{u}=\mathfrak{u}_{1} i+\mathfrak{u}_{2} j+\mathfrak{u}_{3} k$.

We have $\tilde{N}_{\mathfrak{q}_{0}} \in C^{1}$ and we can compute $D \tilde{N}_{\mathfrak{q}_{0}}(0)$ as

$$
D \tilde{N}_{\mathfrak{q}_{0}}(0)=\left.\frac{d}{d t} \tilde{N}_{\mathfrak{q}_{0}}(t \mathfrak{u})\right|_{t=0}=: L_{\mathfrak{q}_{0}}(\mathfrak{u})
$$

where for $\mathfrak{u} \in L^{\frac{2 p}{p-2}} \cap \dot{W}^{1, p} \cap \dot{W}^{1, p^{\prime}}\left(\mathbb{C}, \mathbb{H}_{p}\right)$

$$
\begin{aligned}
L_{\mathfrak{q}_{0}}(\mathfrak{u}) & :=\left(\Pi_{i}\left(\Delta \mathfrak{u}+\partial_{x_{1}}\left[\mathfrak{q}_{0}^{-1} \partial_{x_{1}} \mathfrak{q}_{0} \mathfrak{u}-\mathfrak{u} \mathfrak{q}_{0}^{-1} \partial_{x_{1}} \mathfrak{q}_{0}\right]+\partial_{x_{2}}\left[\mathfrak{q}_{0}^{-1} \partial_{x_{2}} \mathfrak{q}_{0} \mathfrak{u}-\mathfrak{u} \mathfrak{q}_{0}^{-1} \partial_{x_{2}} \mathfrak{q}_{0}\right]\right)\right. \\
& \left.\Pi_{j k}\left(\partial_{x_{1}} \mathfrak{u}-\partial_{x_{2}} \mathfrak{u} i+\left[\mathfrak{q}_{0}^{-1} \partial_{x_{1}} \mathfrak{q}_{0} \mathfrak{u}-\mathfrak{u} \mathfrak{q}_{0}^{-1} \partial_{x_{1}} \mathfrak{q}_{0}\right]-\left[\mathfrak{q}_{0}^{-1} \partial_{x_{2}} \mathfrak{q}_{0} \mathfrak{u}-\mathfrak{u q} \mathfrak{q}_{0}^{-1} \partial_{x_{2}} \mathfrak{q}_{0}\right] i\right)\right)
\end{aligned}
$$

In order to use a fixed-point argument for $\tilde{N}_{\mathfrak{q}_{0}}$, we will show that $L_{\mathfrak{q}_{0}}$ is an isomorphism. More precisely we prove the following lemma.

Lemma III.6. For any $\Theta>0$ there exists a $\varepsilon>0$ so that the following holds for any $\omega_{0}, \mathfrak{g}_{0}$ and $\mathfrak{q}_{0}$ as above.

For any $\omega \in\left(\dot{W}^{-1, p} \cap \dot{W}^{-1, p^{\prime}}\right)(\mathbb{C}, i \mathbb{R}), \mathfrak{g} \in\left(L^{p} \cap L^{p^{\prime}}\right)(\mathbb{C}, \operatorname{span}\{j, k\})$ there exists a unique $\mathfrak{u} \in L^{\frac{2 p}{p-2}} \cap \dot{W}^{1, p} \cap \dot{W}^{1, p^{\prime}}\left(\mathbb{C}, \mathbb{H}_{p}\right)$ so that

$$
(\omega, \mathfrak{g})=L_{\mathfrak{q}_{0}}(\mathfrak{u})
$$

and for some constant $C=C\left(\omega_{0}, \alpha_{0}, \Theta\right)>0$ it holds

$$
\begin{aligned}
\|\mathfrak{u}\|_{L^{\frac{2 p}{p-2}}}+\|\nabla \mathfrak{u}\|_{L^{p}(\mathbb{C})}+\|\nabla \mathfrak{u}\|_{L^{p^{\prime}(\mathbb{C})}} & \leq C\left[\left(\|\omega\|_{\dot{W}^{-1, p}(\mathbb{C})}+\|\omega\|_{\dot{W}^{-1, p^{\prime}}(\mathbb{C})}\right)\right. \\
& \left.+\left(\|\mathfrak{g}\|_{L^{p}(\mathbb{C})}+\|\mathfrak{g}\|_{L^{p^{\prime}(\mathbb{C})}}\right)\right] .
\end{aligned}
$$

${ }^{8}$ Indeed for a Schwartz function one has

$$
\mathfrak{u}(x)=\frac{1}{2 \pi} \int_{\mathbb{C}} \nabla_{x} \log |x-y| \cdot \nabla \mathfrak{u}(y) d y \quad \Rightarrow \quad\|\mathfrak{u}\|_{\infty} \leq(2 \pi)^{-1}\left\|\nabla_{x} \log |x-y|\right\|_{L^{2, \infty}}\|\nabla \mathfrak{u}\|_{L^{2,1}}
$$

Generalized Hölder inequality (see [9]) gives moreover

$$
\|\nabla \mathfrak{u}\|_{L^{2,1}} \leq C\|\nabla \mathfrak{u}\|_{L^{p}}^{\alpha}\|\nabla \mathfrak{u}\|_{L^{p^{\prime}}}^{1-\alpha} .
$$

where $2^{-1}=\alpha p^{-1}+(1-\alpha){p^{\prime}}^{-1}$. If $\mathfrak{u} \in \dot{W}^{1, p} \cap \dot{W}^{1, p^{\prime}} \cap L^{\frac{2 p}{p-2}}$ then $\tilde{\mathfrak{u}}(x)=\frac{1}{2 \pi} \int_{\mathbb{C}} \nabla_{x} \log |x-y| \cdot \nabla \mathfrak{u}(y) d y$ satisfies $\Delta \tilde{\mathfrak{u}}=\Delta \mathfrak{u}$ in $\mathcal{S}^{\prime}(\mathbb{C})$. One has $\|\tilde{\mathfrak{u}}\|_{\infty} \leq(2 \pi)^{-1}\left\|\nabla_{x} \log |x-y|\right\|_{L^{2, \infty}}\|\nabla \mathfrak{u}\|_{L^{2,1}}$ and moreover $\tilde{\mathfrak{u}} \in L^{\frac{2 p}{p-2}}$. $\tilde{\mathfrak{u}}-\mathfrak{u}$ is a harmonic function belonging to $L^{\frac{2 p}{p-2}}$, hence $\tilde{\mathfrak{u}}-\mathfrak{u}=0$. 


\section{Proof of lemma III.6.}

Claim 1. $\mathbf{L}_{1}(\mathfrak{u})$ is invertible $\left(\mathfrak{q}_{0}=1\right)$ as a map

$$
L_{1}:\left(\dot{W}^{1, p^{\prime}} \cap L^{\frac{2 p}{p-2}}\right)\left(\mathbb{C}, \mathbb{H}_{p}\right) \rightarrow\left(\dot{W}^{-1, p^{\prime}}(i \mathbb{R}) \times\left(L^{p} \cap L^{p^{\prime}}\right)(\mathbb{C}, \operatorname{span}\{j, k\})\right)
$$

The operator $L_{1}$ is given by

$$
\begin{aligned}
L_{1}(\mathfrak{u}) & =\frac{d}{d t} \mathbf{N}\left(e^{t \mathfrak{u}}\right)_{t=0}=\left(\Pi_{i}(\Delta \mathfrak{u}), \Pi_{j k}\left(\partial_{x_{1}} \mathfrak{u}-\partial_{x_{2}} \mathfrak{u} i\right)\right) \\
& =\left(\Delta \mathfrak{u}_{1} i,\left(\partial_{x_{1}} \mathfrak{u}_{2}-\partial_{x_{2}} \mathfrak{u}_{3}\right) j+\left(\partial_{x_{1}} \mathfrak{u}_{3}+\partial_{x_{2}} \mathfrak{u}_{2}\right) k\right)
\end{aligned}
$$

Given $f \in \dot{W}^{-1, p^{\prime}}(\mathbb{C}, \mathbb{R}), a, b \in L^{p^{\prime}}(\mathbb{C}, \mathbb{R})$ there is a unique triple $\mathfrak{u}_{1}, \mathfrak{u}_{2}, \mathfrak{u}_{3} \in \dot{W}^{1, p} \cap \dot{W}^{1, p^{\prime}} \cap$ $L^{\frac{2 p}{p-2}}(\mathbb{C}, \mathbb{R})$ such that

$$
L_{1}(\mathfrak{u})=(f i, a j+b k) .
$$

More precisely the following system should be satisfied:

$$
\begin{gathered}
\left\{\begin{array}{l}
\Delta \mathfrak{u}_{1}=f \\
\partial_{z}\left(\mathfrak{u}_{2}-\mathfrak{u}_{3} i\right)=a-b i
\end{array}\right. \\
\mathfrak{u}_{1}(x)=\frac{1}{2 \pi} \log (|x|) * f(x), \quad \mathfrak{u}_{2}-\mathfrak{u}_{3} i=\frac{1}{4 \pi}(a-b i) * \frac{1}{\bar{z}} .
\end{gathered}
$$

Classical estimates give

$$
\left\|\mathfrak{u}_{1}\right\|_{L^{\frac{2 p}{p-2}}}+\left\|\nabla \mathfrak{u}_{1}\right\|_{L^{p^{\prime}}} \lesssim\|f\|_{\dot{W}^{-1, p^{\prime}}}, \quad\left\|\mathfrak{u}_{2}-\mathfrak{u}_{3} i\right\|_{L^{\frac{2 p}{p-2}}}+\left\|\nabla\left(\mathfrak{u}_{2}-\mathfrak{u}_{3} i\right)\right\|_{L^{p^{\prime}}} \lesssim\|a-b i\|_{L^{p^{\prime}}}
$$

\section{The Claim 1 is proved.}

Observe that

$$
\begin{gathered}
L_{\mathfrak{q}_{0}}(\mathfrak{u})-L_{1}(\mathfrak{u})=\left(\Pi_{i}\left(\partial_{x_{1}}\left[\mathfrak{q}_{0}^{-1} \partial_{x_{1}} \mathfrak{q}_{0} \mathfrak{u}-\mathfrak{u} \mathfrak{q}_{0}^{-1} \partial_{x_{1}} \mathfrak{q}_{0}\right]+\partial_{x_{2}}\left[\mathfrak{q}_{0}^{-1} \partial_{x_{2}} \mathfrak{q}_{0} \mathfrak{u}-\mathfrak{u q _ { 0 } ^ { - 1 }} \partial_{x_{2}} \mathfrak{q}_{0}\right]\right)\right. \\
\left.\Pi_{j k}\left(\left[\mathfrak{q}_{0}^{-1} \partial_{x_{1}} \mathfrak{q}_{0} \mathfrak{u}-\mathfrak{u} \mathfrak{q}_{0}^{-1} \partial_{x_{1}} \mathfrak{q}_{0}\right]-\left[\mathfrak{q}_{0}^{-1} \partial_{x_{2}} \mathfrak{q}_{0} \mathfrak{u}-\mathfrak{u} \mathfrak{q}_{0}^{-1} \partial_{x_{2}} \mathfrak{q}_{0}\right] i\right)\right)
\end{gathered}
$$

We have

$$
\begin{aligned}
& \left\|\partial_{x_{\ell}}\left(\mathfrak{q}_{0}^{-1} \partial_{x_{\ell}} \mathfrak{q}_{0} \mathfrak{u}-\mathfrak{u} \mathfrak{q}_{0}^{-1} \partial_{x_{\ell}} \mathfrak{q}_{0}\right)\right\|_{\dot{W}^{-1, p^{\prime}}} \leq\left\|\mathfrak{q}_{0}^{-1} \partial_{x_{\ell}} \mathfrak{q}_{0} \mathfrak{u}-\mathfrak{u} \mathfrak{q}_{0}^{-1} \partial_{x_{\ell}} \mathfrak{q}_{0}\right\|_{L^{p^{\prime}}} \\
& \quad \leq\left\|\nabla \mathfrak{q}_{0}\right\|_{L^{2}}\|\mathfrak{u}\|_{L^{\frac{2 p}{p-2}}} \leq \varepsilon \Theta\|\mathfrak{u}\|_{L^{\frac{2 p}{p-2}}}
\end{aligned}
$$

Choosing $\varepsilon>0$ small enough (depending on $\Theta$ ) we obtain that $L_{\mathfrak{q}_{0}}$ is an invertible map from $\dot{W}^{1, p^{\prime}} \cap L^{\frac{2 p}{p-2}}$ to $\dot{W}^{-1, p^{\prime}}(\mathbb{C}, i \mathbb{R}) \times L^{p^{\prime}}(\mathbb{C}, \operatorname{span}\{j, k\})$, 
Claim 2. Assuming now $\omega \in\left(\dot{W}^{-1, p} \cap \dot{W}^{-1, p^{\prime}}\right)(\mathbb{C}, i \mathbb{R}), \mathfrak{g} \in\left(L^{p} \cap L^{p^{\prime}}\right)(\mathbb{C}, \operatorname{span}\{j, k\})$ we prove that the unique solution $\mathfrak{u}$ of $L_{\mathfrak{q}_{0}}(\mathfrak{u})$ is in $\dot{W}^{1, p}$.

From the fact that $(w, \mathfrak{g})=L_{\mathfrak{q}_{0}}$ it follows:

$$
\left\{\begin{aligned}
\Delta \mathfrak{u}_{1} i=\omega- & \Pi_{i}\left(\partial_{x_{1}}\left(\mathfrak{q}_{0}^{-1} \partial_{x_{1}} \mathfrak{q}_{0} \mathfrak{u}-\mathfrak{u} \mathfrak{q}_{0}^{-1} \partial_{x_{1}} \mathfrak{q}_{0}\right)\right)-\Pi_{i}\left(\partial_{x_{2}}\left(\mathfrak{q}_{0}^{-1} \partial_{x_{2}} \mathfrak{q}_{0} \mathfrak{u}-\mathfrak{u} \mathfrak{q}_{0}^{-1} \partial_{x_{2}} \mathfrak{q}_{0}\right)\right) \\
& \left(\partial_{x_{1}} \mathfrak{u}_{2}-\partial_{x_{2}} \mathfrak{u}_{3}\right) j+\left(\partial_{x_{1}} \mathfrak{u}_{3}+\partial_{x_{2}} \mathfrak{u}_{2}\right) k=\mathfrak{g} \\
+ & \Pi_{j k}\left(-\left[\mathfrak{q}_{0}^{-1} \partial_{x_{1}} \mathfrak{q}_{0} \mathfrak{u}-\mathfrak{u} \mathfrak{q}_{0}^{-1} \partial_{x_{1}} \mathfrak{q}_{0}\right]+\left[\mathfrak{q}_{0}^{-1} \partial_{x_{2}} \mathfrak{q}_{0} \mathfrak{u}-\mathfrak{u} \mathfrak{q}_{0}^{-1} \partial_{x_{2}} \mathfrak{q}_{0}\right] i\right)
\end{aligned}\right.
$$

We observe that

$$
\left(\partial_{x_{1}} \mathfrak{u}_{2}-\partial_{x_{2}} \mathfrak{u}_{3}\right) j+\left(\partial_{x_{1}} \mathfrak{u}_{3}+\partial_{x_{2}} \mathfrak{u}_{2}\right) k=2 \partial_{\bar{z}}\left(\mathfrak{u}_{2}+\mathfrak{u}_{3} i\right) j
$$

Therefore we can write the second equation in ([II.106) in the following way:

$2 \partial_{\bar{z}}\left(\mathfrak{u}_{2}+\mathfrak{u}_{3} i\right)=-\mathfrak{g} j+\Pi_{j k}\left[\mathfrak{q}_{0}^{-1} \partial_{x_{1}} \mathfrak{q}_{0} \mathfrak{u}-\mathfrak{u} \mathfrak{q}_{0}^{-1} \partial_{x_{1}} \mathfrak{q}_{0}\right] j+\left(\Pi_{j k}\left(\left[\mathfrak{q}_{0}^{-1} \partial_{x_{2}} \mathfrak{q}_{0} \mathfrak{u}-\mathfrak{u} \mathfrak{q}_{0}^{-1} \partial_{x_{2}} \mathfrak{q}_{0}\right] i\right) j\right.$

Let $p^{\prime}<r<2$, since $\nabla \mathfrak{q}_{0} \in L^{p}$ we have for $\ell=1,2$

$$
\left\|\mathfrak{q}_{0}^{-1} \partial_{x_{\ell}} \mathfrak{q}_{0} \mathfrak{u}\right\|_{L^{t}} \lesssim\left\|\nabla \mathfrak{q}_{0}\right\|_{L^{p}}\|\mathfrak{u}\|_{L^{\frac{2 r}{2-r}}}
$$

for $\frac{1}{t}=\frac{1}{p}+\frac{2-r}{2 r}$. Observe that $p>t>2$, since $r>p^{\prime}$.

From (III.106) and (III.108) it follows $\nabla \mathfrak{u} \in L^{t}$. We have also $\nabla \mathfrak{u} \in L^{t^{\prime}}$. This implies that $\mathfrak{u} \in L^{\infty}$ (see previous footnote). Therefore

$$
\left\|\mathfrak{q}_{0}^{-1} \partial_{x_{\ell}} \mathfrak{q}_{0} \mathfrak{u}\right\|_{L^{p}} \lesssim\left\|\nabla \mathfrak{q}_{0}\right\|_{L^{p}}\|\mathfrak{u}\|_{\infty}
$$

From (III.106) it follows that $\nabla \mathfrak{u} \in L^{p}$ and the Claim 2 is proved. This concludes the proof of lemma III.6.

\section{Proof of proposition III.3 continued.}

For $\varepsilon=\varepsilon(\Theta)>0$ chosen small enough, and for any $\left(\omega_{0}, \mathfrak{g}_{0}\right) \in \mathcal{V}_{\varepsilon, \Theta}$ the local inversion theorem applied to $\mathbf{N}$ gives the existence of some $\delta>0$ (that might depend on $\left(\omega_{0}, \mathfrak{g}_{0}\right)$ ) such that, for every $(\omega, \mathfrak{g}) \in \mathcal{U}_{\varepsilon}$ with

$$
\begin{aligned}
& \left\|\omega-\omega_{0}\right\|_{\dot{W}^{-1, p}(\mathbb{C})}+\left\|\omega-\omega_{0}\right\|_{\dot{W}^{-1, p^{\prime}}(\mathbb{C})}<\delta \\
& \left\|\mathfrak{g}-\mathfrak{g}_{0}\right\|_{L^{p}(\mathbb{C})}+\left\|\mathfrak{g}-\mathfrak{g}_{0}\right\|_{L^{p^{\prime}}(\mathbb{C})}<\delta,
\end{aligned}
$$

we find $\mathfrak{q}=\mathfrak{q}_{0} e^{\mathfrak{u}} \in \dot{W}^{1, p} \cap \dot{W}^{1, p^{\prime}}(\mathbb{C}, \mathcal{U}(\mathbb{H}))$, so that $\mathfrak{q}-1 \in L^{\frac{2 p}{p-2}}(\mathbb{C}, \mathbb{H})$ and (III.94) is satisfied. 
It remains to prove (III.97), (III.98) and (III.99). The local inversion theorem does not imply the estimates (III.97), (III.98) and (III.99). Anyway for every $\omega, \mathfrak{g} \in \mathcal{U}_{\varepsilon}$ satisfying (III.110) and (III.111) and for every $\sigma$ we can choose $\varepsilon$ and $\delta$ small enough so that

$$
\|\nabla \mathfrak{q}\|_{L^{2}(\mathbb{C})} \leq \sigma .
$$

The next lemma shows that if $\sigma$ is small enough then (III.97), (III.98) and (III.99) hold for a uniform constant $\Theta$.

Lemma III.7. There exists $a \Theta>0$ and $a \sigma>0$ so that whenever $\mathfrak{q} \in \dot{W}^{1, p} \cap$ $\dot{W}^{1, p^{\prime}}\left(\mathbb{C},(\mathcal{U}(\mathbb{H}))\right.$ and $\mathfrak{q}-1 \in L^{\frac{2 p}{p-2}}(\mathbb{C}, \mathbb{H})$ so that $(\underline{\text { III.94 }})$ is satisfied and it holds

$$
\|\nabla \mathfrak{q}\|_{L^{2}(\mathbb{C})} \leq \sigma
$$

then (III.97), (III.98) and (III.99) hold.

\section{Proof of Lemma III.7.}

Let us write $\omega=\Delta \mu$ with $\nabla \mu \in L^{p} \cap L^{p^{\prime}}$. Let $\xi \in \dot{W}^{1, p} \cap \dot{W}^{1, p^{\prime}}(\mathbb{C}, \mathbb{R})$ be such that

$$
\left\{\begin{array}{c}
\Pi_{i}\left(\mathfrak{q}^{-1} \partial_{x_{1}} \mathfrak{q}-\partial_{x_{1}} \mu\right)=-\partial_{x_{2}} \xi i \\
\Pi_{i}\left(\mathfrak{q}^{-1} \partial_{x_{2}} \mathfrak{q}-\partial_{x_{2}} \mu\right)=\partial_{x_{1}} \xi i
\end{array}\right.
$$

Then

$$
-\Delta \xi i=\Pi_{i}\left(\partial_{x_{2}}\left(\mathfrak{q}^{-1} \partial_{x_{1}} \mathfrak{q}\right)\right)-\Pi_{i}\left(\partial_{x_{1}}\left(\mathfrak{q}^{-1} \partial_{x_{2}} \mathfrak{q}\right)\right) .
$$

For every $q \in\left[p^{\prime}, p\right]$ it holds 9

$$
\|\nabla \xi\|_{L^{q}} \lesssim\|\nabla \mathfrak{q}\|_{L^{2, \infty}}\|\nabla \mathfrak{q}\|_{L^{q}} \lesssim \sigma\|\nabla \mathfrak{q}\|_{L^{q}}
$$

We can write

$$
\begin{aligned}
\mathfrak{q}^{-1} \partial_{x_{1}} \mathfrak{q}-\mathfrak{q}^{-1} \partial_{x_{2}} \mathfrak{q} i & =\Pi_{i}\left(\mathfrak{q}^{-1} \partial_{x_{1}} \mathfrak{q}\right)-\Pi_{i}\left(\mathfrak{q}^{-1} \partial_{x_{2}} \mathfrak{q}\right) i \\
& +\Pi_{j k}\left(\mathfrak{q}^{-1} \partial_{x_{1}} \mathfrak{q}\right)-\Pi_{j k}\left(\mathfrak{q}^{-1} \partial_{x_{2}} \mathfrak{q}\right) i \\
& =\left(\partial_{x_{1}} \xi-\partial_{x_{2}} \xi i\right)+\Pi_{i}\left(\partial_{x_{1}} \mu-\partial_{x_{2}} \mu\right)+\mathfrak{g}
\end{aligned}
$$

or equivalently

$$
\partial_{x_{1}} \mathfrak{q}-\partial_{x_{2}} \mathfrak{q} i=\mathfrak{q}\left(\left(\partial_{x_{1}} \xi-\partial_{x_{2}} \xi i\right)+\Pi_{i}\left(\partial_{x_{1}} \mu-\partial_{x_{2}} \mu\right)+\mathfrak{g}\right) .
$$

Therefore by combining (III.116) and (III.118) we get for every $q \in\left[p^{\prime}, p\right]$

\footnotetext{
${ }^{9}$ We use the fact that if $\nabla a \in L^{2, \infty}, \nabla b \in L^{q}$, with $q \in\left[p^{\prime}, p\right]$ and if $-\Delta \phi=\nabla a \cdot \nabla^{\perp} b$, in $\mathbb{C}$ then $\nabla \phi \in L^{q}$ with $\|\nabla \phi\|_{L^{q}} \leq C_{q}\|\nabla b\|_{L^{q}}\|\nabla a\|_{L^{2, \infty}}$. The constant $C_{q}$ is uniformly bounded if $q \in\left[p^{\prime}, p\right]$ (see [10]).
} 


$$
\begin{aligned}
\|\nabla \mathfrak{q}\|_{L^{q}} & \leq C\left[\mid \nabla \xi\left\|_{L^{q}}+\right\| \nabla \mu\left\|_{L^{q}}+\right\| \mathfrak{g} \|_{L^{q}}\right] \\
& \leq C \sigma\|\nabla \mathfrak{q}\|_{L^{q}}+C\|\omega\|_{\dot{W}^{-1, q}}+C\|\mathfrak{g}\|_{L^{q}} .
\end{aligned}
$$

Choosing $\Theta=\frac{C}{1-C \sigma}$ we have

$$
\|\nabla \mathfrak{q}\|_{L^{q}} \leq \Theta\left(\|\omega\|_{\dot{W}^{-1, q}}+\|\mathfrak{g}\|_{L^{q}}\right) .
$$

This concludes the proof of lemma III.7.

End of the proof of Proposition III.3. As we have already observed if $\varepsilon$ is small enough the fact that $\omega, \mathfrak{g} \in \mathcal{U}_{\varepsilon}$ implies that $\mathfrak{q} \in \mathcal{V}_{\varepsilon, \Theta}$, it satisfies $\|\nabla \mathfrak{q}\|_{L^{2}} \leq \sigma$ where $\sigma$ is the constant appearing in Lemma III.7. Therefore thanks to lemma \II.7 the openness property (iv) is proven and Proposition 111.3 is then established.

\section{Proof of Theorem 世I.7.}

Let $\mathfrak{f}$ solve (III.91) with $\mathfrak{q} \in N^{-1}\left(0,-\partial_{x_{1}} \alpha j+\partial_{x_{2}} \alpha k\right)$ and $\|\nabla \mathfrak{q}\|_{L^{2}} \leq \Theta\|\nabla \alpha\|_{L^{2}}$. By definition $\mathfrak{q}$ satisfies

$$
\left\{\begin{array}{c}
\Pi_{i}\left(\partial_{x_{1}}\left(\mathfrak{q}^{-1} \partial_{x_{1}} \mathfrak{q}\right)+\partial_{x_{2}}\left(\mathfrak{q}^{-1} \partial_{x_{2}} \mathfrak{q}\right)\right)=0 \\
\Pi_{j k}\left(\mathfrak{q}^{-1} \partial_{x_{1}} \mathfrak{q}-\mathfrak{q}^{-1} \partial_{x_{2}} \mathfrak{q} i\right)=-\partial_{x_{1}} \alpha j+\partial_{x_{2}} \alpha k .
\end{array}\right.
$$

We analyze the first equation in (III.120).

We have

$$
\left\{\begin{array}{c}
\Pi_{i}\left(\partial_{x_{1}}\left(\mathfrak{q}^{-1} \partial_{x_{1}} \mathfrak{q}\right)+\partial_{x_{2}}\left(\mathfrak{q}^{-1} \partial_{x_{2}} \mathfrak{q}\right)\right)=0 \\
\mathbb{\mathbb { }} \\
\partial_{x_{1}}\left(\Pi_{i}\left(\mathfrak{q}^{-1} \partial_{x_{1}} \mathfrak{q}\right)\right)+\partial_{x_{2}}\left(\Pi_{i}\left(\mathfrak{q}^{-1} \partial_{x_{2}} \mathfrak{q}\right)\right)=0 .
\end{array}\right.
$$

Therefore there exists $\zeta \in \dot{W}^{1,2}(\mathbb{C}, i \mathbb{R})$ such that

$$
\left\{\begin{array}{c}
\Pi_{i}\left(\mathfrak{q}^{-1} \partial_{x_{1}} \mathfrak{q}\right)=-\partial_{x_{2}} \zeta \\
\Pi_{i}\left(\mathfrak{q}^{-1} \partial_{x_{2}} \mathfrak{q}\right)=\partial_{x_{1}} \zeta
\end{array}\right.
$$

From (III.122) it follows in particular that

$$
\begin{aligned}
-\Delta \zeta & =\partial_{x_{2}}\left(\Pi_{i}\left(\mathfrak{q}^{-1} \partial_{x_{1}} \mathfrak{q}\right)\right)-\partial_{x_{1}}\left(\Pi_{i}\left(\mathfrak{q}^{-1} \partial_{x_{2}} \mathfrak{q}\right)\right) \\
& =\Pi_{i}\left(\partial_{x_{2}}\left(\mathfrak{q}^{-1} \partial_{x_{1}} \mathfrak{q}\right)-\partial_{x_{1}}\left(\mathfrak{q}^{-1} \partial_{x_{2}} \mathfrak{q}\right)\right) .
\end{aligned}
$$


The right hand side of (III.124) is a sum of jacobians, hence it is in the Hardy space $\mathcal{H}^{1}(\mathbb{C})$. It follows in particular that $\nabla \zeta \in L^{2,1}(\mathbb{C})$, with

$$
\|\nabla \zeta\|_{L^{2,1}} \lesssim\|\nabla \mathfrak{q}\|_{L^{2}}^{2}
$$

We have

$$
\begin{aligned}
\mathfrak{q}^{-1} \partial_{x_{1}} \mathfrak{q}-\mathfrak{q}^{-1} \partial_{x_{2}} \mathfrak{q} i & =\Pi_{i}\left(\mathfrak{q}^{-1} \partial_{x_{1}} \mathfrak{q}\right)+\Pi_{j k}\left(\mathfrak{q}^{-1} \partial_{x_{1}} \mathfrak{q}\right)-\Pi_{i}\left(\mathfrak{q}^{-1} \partial_{x_{2}} \mathfrak{q}\right) i-\Pi_{j k}\left(\mathfrak{q}^{-1} \partial_{x_{2}} \mathfrak{q}\right) i \\
& =-\partial_{x_{2}} \zeta-\partial_{x_{1}} \zeta i+\Pi_{j k}\left(\mathfrak{q}^{-1} \partial_{x_{1}} \mathfrak{q}-\mathfrak{q}^{-1} \partial_{x_{2}} \mathfrak{q} i\right) \\
& =-2 i\left(\partial_{z} \zeta\right)-\partial_{x_{1}} \alpha j+\partial_{x_{2}} \alpha k
\end{aligned}
$$

In (III.124) we use the fact that $\Pi_{j k}(a i)=\Pi_{j k}(a) i$ for $a \in \mathbb{H}$. By combining (III.91), (III.120) and (III.124) we get

$$
\partial_{x_{1}}[\mathfrak{q} \mathfrak{f}]-\partial_{x_{2}}[\mathfrak{q} i \mathfrak{f}]=-2 \mathfrak{q} i\left[\partial_{z} \zeta\right] \mathfrak{f}
$$

We set

$$
-\Delta A=2 \mathfrak{q} i\left[\partial_{z} \zeta\right] \mathfrak{f}
$$

Observe that

$$
\begin{aligned}
\|\nabla A\|_{L^{2, \infty}} & \lesssim\|\mathfrak{q} \mathfrak{f} \nabla \zeta\|_{L^{1}} \lesssim\|\nabla \zeta\|_{L^{2,1}}\|\mathfrak{q} \mathfrak{f}\|_{L^{2, \infty}} \\
& \lesssim\|\nabla \mathfrak{q}\|_{L^{2}}^{2}\|\mathfrak{f}\|_{L^{2, \infty}} \lesssim \varepsilon_{0}^{2}\|\mathfrak{q} \mathfrak{f}\|_{L^{2, \infty}}
\end{aligned}
$$

Since

$$
\partial_{x_{1}}\left(\mathfrak{q} \mathfrak{f}-\partial_{x_{1}} A\right)-\partial_{x_{2}}\left(\mathfrak{q} i \mathfrak{f}+\partial_{x_{2}} A\right)=0,
$$

there exists $B \in \dot{W}^{1,(2, \infty)}$ such that

$$
\left\{\begin{array}{c}
\mathfrak{q} \mathfrak{f}-\partial_{x_{1}} A=-\partial_{x_{2}} B \\
-\mathfrak{q} i \mathfrak{f}-\partial_{x_{2}} A=\partial_{x_{1}} B .
\end{array}\right.
$$

Therefore

$$
\left\{\begin{array}{c}
\mathfrak{f}=\mathfrak{q}^{-1}\left(\partial_{x_{1}} A-\partial_{x_{2}} B\right) \\
\mathfrak{f}=i \mathfrak{q}^{-1}\left(\partial_{x_{2}} A+\partial_{x_{1}} B\right) .
\end{array}\right.
$$


From (III.129) it follows

$$
\left\{\begin{array}{l}
\partial_{x_{1}} A-\partial_{x_{2}} B=\mathfrak{q} i \mathfrak{q}^{-1}\left(\partial_{x_{2}} A+\partial_{x_{1}} B\right) \\
-\partial_{x_{2}} A-\partial_{x_{1}} B=\mathfrak{q} i \mathfrak{q}^{-1}\left(\partial_{x_{1}} A-\partial_{x_{2}} B\right) \\
-\partial_{x_{2}} B=\mathfrak{q} i \mathfrak{q}^{-1}\left(\partial_{x_{2}} A+\partial_{x_{1}} B\right)-\partial_{x_{1}} A \\
-\partial_{x_{1}} B=\mathfrak{q} i \mathfrak{q}^{-1}\left(\partial_{x_{1}} A-\partial_{x_{2}} B\right)+\partial_{x_{2}} A \\
-\Delta B=\partial_{x_{1}}\left(\mathfrak{q} i \mathfrak{q}^{-1}\left(\partial_{x_{1}} A-\partial_{x_{2}} B\right)\right)+\partial_{x_{2}}\left(\mathfrak{q} i \mathfrak{q}^{-1}\left(\partial_{x_{2}} A+\partial_{x_{1}} B\right)\right)
\end{array}\right.
$$

We observe that $-\partial_{x_{1}}\left[\mathfrak{q} i \mathfrak{q}^{-1} \partial_{x_{2}} B\right]+\partial_{x_{2}}\left[\mathfrak{q} i \mathfrak{q}^{-1} \partial_{x_{1}} B\right]$ is sum of Jacobians and therefore we can apply Wente's Lemma (case $\left.L^{2}-L^{2, \infty}\right)$ :

$$
\begin{aligned}
\|\nabla B\|_{L^{2, \infty}} & \lesssim\|\nabla A\|_{L^{2, \infty}}+\|\nabla \mathfrak{q}\|_{L^{2}}\|\nabla B\|_{L^{2, \infty}} \\
& \lesssim\|\nabla A\|_{L^{2, \infty}}+\varepsilon_{0}\|\nabla B\|_{L^{2, \infty}}
\end{aligned}
$$

Estimate (III.131) implies that

$$
\begin{aligned}
\|\nabla B\|_{L^{2, \infty}} & \lesssim\|\nabla A\|_{L^{2, \infty}} \\
\|\mathfrak{q} \mathfrak{f}\|_{L^{2, \infty}} & \lesssim\|\nabla A\|_{L^{2, \infty}}+\|\nabla B\|_{L^{2, \infty}} \\
& \lesssim \varepsilon_{0}^{2}\|\mathfrak{q} \mathfrak{f}\|_{L^{2, \infty}} .
\end{aligned}
$$

If $\varepsilon_{0}$ is small enough then $\mathfrak{f} \equiv 0$. This concludes the proof of Theorem III.7.

\section{III.3 Morrey-Type Estimates}

In this section we prove Morrey-type estimates for solutions to (III.43) in the case $n=2$.

Proposition III.4. Let $S \in W^{1,2}(\mathbb{C}, O(2))$ with $S^{2}=I d$ and $u \in L^{2}(\mathbb{C})$ be a solution of (III.43). Then $u \in W_{l o c}^{1, p}$ for every $p \in[1,2)$.

Proof of Proposition III.4. Step 1. Assume that $\|\nabla S\|_{L^{2}(B(0,1))} \leq \varepsilon_{0}$.

Claim: There is $0<\varepsilon_{0}<1$ and $\tilde{S} \in \dot{W}^{1,2}(\mathbb{C}, \operatorname{Sym}(2))$ with $\tilde{S}^{2}=I d$ such that $\tilde{S}=S$ in $B(0,1)$ and $\|\nabla \tilde{S}\|_{L^{2}\left(\mathbb{R}^{2}\right)} \leq C\|\nabla S\|_{L^{2}(B(0,1))}$.

For the proof of the claim we refer to [17].

Now let $v \in L^{2}\left(\mathbb{R}^{2}\right)$ be such that

$$
\nabla^{\perp} v=S \nabla u \text { in } \mathcal{S}^{\prime}\left(\mathbb{R}^{2}\right) .
$$


By arguing as in the previous section we can find $\mathfrak{q} \in \dot{W}^{1,2}(\mathbb{C}, \mathcal{U}(\mathbb{H}))$ with $\|\nabla \mathfrak{q}\|_{L^{2}(\mathbb{C})} \leq$ $C\|\nabla \tilde{S}\|_{L^{2}(\mathbb{C})}$ and $\zeta \in \dot{W}^{1,(2,1)}(\mathbb{C})$ with $\left\|\partial_{z} \zeta\right\|_{L^{2,1}(\mathbb{C})} \leq \varepsilon_{0}^{2}$ such that

$$
\partial_{x_{1}}[\mathfrak{q} \mathfrak{f}]-\partial_{x_{2}}[\mathfrak{q} i \mathfrak{f}]=-2 \mathfrak{q} i\left[\partial_{z} \zeta\right] \mathfrak{f} \text { in } \mathcal{D}^{\prime}(B(0,1))
$$

1. Let $x \in B(0,1 / 2)$ and $0<r<1 / 4$. We consider

$$
\left\{\begin{array}{cc}
-\Delta A=2 \mathfrak{q} i\left[\partial_{z} \zeta\right] \mathfrak{f} & \text { in } B(x, r) \\
A=0 & \text { on } \partial B(x, r) .
\end{array}\right.
$$

We have

$$
\|\nabla A\|_{L^{2, \infty}(B(x, r))} \lesssim \varepsilon_{0}^{2}\|f\|_{L^{2, \infty}(B(x, r))}
$$

2. Since

$$
\partial_{x_{1}}\left(\mathfrak{q} \mathfrak{f}-\partial_{x_{1}} A\right)-\partial_{x_{2}}\left(\mathfrak{q} i \mathfrak{f}+\partial_{x_{2}} A\right)=0,
$$

there exists $B \in \dot{W}^{1,(2, \infty)}(B(x, r))$ such that

$$
\left\{\begin{array}{r}
\mathfrak{q} \mathfrak{f}-\partial_{x_{1}} A=-\partial_{x_{2}} B \\
-\mathfrak{q} i \mathfrak{f}-\partial_{x_{2}} A=\partial_{x_{1}} B
\end{array}\right.
$$

We have

$$
-\Delta B=\partial_{x_{1}}\left(\mathfrak{q} i \mathfrak{q}^{-1}\left(\partial_{x_{1}} A-\partial_{x_{2}} B\right)\right)+\partial_{x_{2}}\left(\mathfrak{q} i \mathfrak{q}^{-1}\left(\partial_{x_{2}} A+\partial_{x_{1}} B\right)\right) \text { in } \mathcal{D}^{\prime}(B(x, r))
$$

We decompose $B=\beta_{1}+\beta_{2}$ in $B(x, r)$ where $\beta_{1}$ and $\beta_{2}$ satisfy respectively

$$
\left\{\begin{array} { c c } 
{ \Delta \beta _ { 1 } = 0 } & { \text { in } B ( 0 , r ) } \\
{ \beta _ { 1 } = B } & { \text { on } \partial B ( 0 , r ) }
\end{array} \text { and } \left\{\begin{array}{cc}
\Delta \beta_{2}=\Delta B & \text { in } B(0, r) \\
\beta_{2}=0 & \text { on } \partial B(0, r)
\end{array}\right.\right.
$$

The following estimates hold:

\section{Estimate of $\beta_{2}$ :}

Wente inequality $\left(L^{2, \infty}-L^{2}\right.$ case) combined with classical Calderon Zygmund inequalities give

$$
\left\|\nabla \beta_{2}\right\|_{L^{2, \infty}(B(x, r))} \leq\|\nabla A\|_{L^{2, \infty}(B(x, r))}+\varepsilon_{0}\|\nabla B\|_{L^{2, \infty}(B(x, r))} .
$$

Estimate of $\beta_{1}$ :

Since $\beta_{1}$ is harmonic, for every $0<\delta<\frac{3}{4}$ we have

$$
\begin{aligned}
\left\|\nabla \beta_{1}\right\|_{L^{2, \infty}(B(x, \delta r))}^{2} & \leq\left\|\nabla \beta_{1}\right\|_{L^{2}(B(x, \delta r))}^{2} \\
& \leq\left(\frac{4 \delta}{3}\right)^{2}\left\|\nabla \beta_{1}\right\|_{L^{2}(B(x, 3 / 4 r))}^{2} \leq C\left(\frac{4 \delta}{3}\right)^{2}\left\|\nabla \beta_{1}\right\|_{L^{2, \infty}(B(x, r))}^{2}
\end{aligned}
$$


where $C$ is a constant independent of $r$. In (III.140) we use the fact that the $L^{2, \infty}$ of the gradient of a harmonic function on the ball $B(x, r)$ controls all its other norms in balls $B(x, \eta r)$ with $\eta<3 / 4$.

\section{Estimate of $B$ :}

Combining the previous estimates we obtain

$$
\begin{aligned}
\|\nabla B\|_{L^{2, \infty}(B(x, \delta r))} & \lesssim\left\|\nabla \beta_{1}\right\|_{L^{2, \infty}(B(x, \delta r)}+\left\|\nabla \beta_{2}\right\|_{L^{2, \infty}(B(x, \delta r)} \\
& \lesssim\left(\frac{4 \delta}{3}\right)\left\|\nabla \beta_{1}\right\|_{L^{2, \infty}(B(x, r)}+\|\nabla A\|_{L^{2, \infty}(B(x, r))}+\varepsilon_{0}\|\nabla B\|_{L^{2, \infty}(B(x, r))} \\
& \lesssim\left(\frac{4 \delta}{3}\right)\left[\left\|\nabla \beta_{2}\right\|_{L^{2, \infty}(B(x, r)}+\|\nabla B\|_{L^{2, \infty}(B(x, r))}\right] \\
& +\|\nabla A\|_{L^{2, \infty}(B(x, r))}+\varepsilon_{0}\|\nabla B\|_{L^{2, \infty}(B(x, r))} \\
& \lesssim\left(\frac{4 \delta}{3}\right)\left[\|\nabla A\|_{L^{2, \infty}(B(x, r))}+\varepsilon_{0}\|\nabla B\|_{L^{2, \infty}(B(x, r))}+\|\nabla B\|_{L^{2, \infty}(B(x, r))}\right] \\
& +\|\nabla A\|_{L^{2, \infty}(B(x, r))}+\varepsilon_{0}\|\nabla B\|_{L^{2, \infty}(B(x, r))} \\
& \lesssim\left[\left(\frac{4 \delta}{3}\right) \varepsilon_{0}^{2}+\varepsilon_{0}^{2}\right]\|\mathfrak{f}\|_{L^{2, \infty}(B(0, r))} \\
& +\left[\left(\frac{4 \delta}{3}\right) \varepsilon_{0}+\left(\frac{4 \delta}{3}\right)+\varepsilon_{0}\right]\|\nabla B\|_{L^{2, \infty}(B(x, r))}
\end{aligned}
$$

Since $\|\nabla B\|_{L^{2, \infty}(B(x, r))} \leq\|\nabla A\|_{L^{2, \infty}(B(x, r))}+\|\mathfrak{f}\|_{L^{2, \infty}(B(x, r))}$ from (III.141) one deduces that

$$
\begin{aligned}
\|\nabla B\|_{L^{2, \infty}(B(x, \delta r))} & \lesssim\left[\left(\frac{4 \delta}{3}\right) \varepsilon_{0}^{2}+\varepsilon_{0}^{2}\right]\|\mathfrak{f}\|_{L^{2, \infty}(B(0, r))} \\
& +\left[\left(\frac{4 \delta}{3}\right) \varepsilon_{0}+\left(\frac{4 \delta}{3}+\varepsilon_{0}\right)\right]\left(1+\varepsilon_{0}^{2}\right)\|\mathfrak{f}\|_{L^{2, \infty}(B(0, r))} .
\end{aligned}
$$

By combining (III.136) and (III.142) we obtain

$$
\begin{aligned}
\|\mathfrak{f}\|_{L^{2, \infty}(B(x, \delta r))} & \lesssim\|\nabla A\|_{L^{2, \infty}(B(x, \delta r))}+\|\nabla B\|_{L^{2, \infty}(B(x, \delta r))} \\
& \lesssim \gamma\|\mathfrak{f}\|_{L^{2, \infty}(B(0, r))} .
\end{aligned}
$$

where $\gamma=\gamma\left(\delta, \varepsilon_{0}\right)<1$. By iterating (III.143) we get the existence of a constant $0<\alpha<1$ such that

$$
\sup _{x \in B(0,1 / 2), 0<r<1 / 4} r^{-\alpha}\|f\|_{L^{2, \infty}(B(x, r))}<+\infty .
$$

Now we plug the estimate (III.144) into (III.134) and we get

$$
\sup _{x \in B(0,1 / 2), 0<r<1 / 4} r^{-\alpha}\|\Delta A\|_{L^{1}(B(x, r))}<+\infty .
$$


and therefore

$$
\sup _{x \in B(0,1 / 2), 0<r<1 / 4} r^{-\alpha}\|\nabla A\|_{L^{2, \infty}(B(x, r))}<+\infty .
$$

From (III.145) it follows in particular that $\nabla A \in L^{q}(B(0,1 / 4))$ for all $q<\frac{2-\alpha}{1-\alpha}$ (See again Adams [1], Remark after Proposition 3.2).

From (III.136), (III.144), (III.146) it follows that

$$
\sup _{x \in B(0,1 / 2), 0<r<1 / 4} r^{-\alpha}\|\nabla B\|_{L^{2, \infty}(B(x, r))}<+\infty .
$$

By plugging (III.147) into (III.137) and (III.138) one gets that

$$
\sup _{x \in B(0,1 / 2), 0<r<1 / 4} r^{-\alpha}\|\Delta B\|_{L^{2, \infty}(B(x, r))}<+\infty .
$$

which implies that $\nabla B \in L^{q}(B(0,1 / 4))$ for all $q<\frac{2-\alpha}{1-\alpha}$ as well. Therefore $\mathfrak{f} \in L^{q}(B(0,1 / 4))$ for all $q<\frac{2-\alpha}{1-\alpha}$ as well. Actually one can show by bootstrap arguments that $\mathfrak{f} \in L_{\text {loc }}^{q}$ for all $q<+\infty$.

Step 2. From Step 1 it follows that $S u \in L_{l o c}^{q}(\mathbb{C})$ for all $q<+\infty$. Since $u$ solves (III.43) we have

$$
\Delta(S u)=\operatorname{div}(\nabla(S u))=\operatorname{div}(\nabla S S S u) \text { in } \mathcal{D}^{\prime}(\mathbb{C}) .
$$

From (III.149) one gets that $\nabla(S u) \in L_{l o c}^{\frac{2 q}{q+2}}$ for all $q<+\infty$ and therefore $\nabla u=\nabla S(S u)+$ $S \nabla(S u) \in L_{l o c}^{p}$ for all $p<2$. This concludes the proof of proposition III.4 which itself implies theorem $\mathrm{I} .1$ in the case of $2-\mathrm{D}$ codomains.

\section{Proof of theorem I.1 : the general case $n \geq 2$}

We are going to present here another approach to study the regularity of the equation (III.43) which works for every $n \geq 2$. We start by showing the bootstrap test:

Theorem IV.8. Let $S \in \dot{W}^{1,2}(\mathbb{C}, O(n))$ with $S^{2}=I d$ and $u \in L^{2}\left(\mathbb{C}, \mathbb{R}^{n}\right)$ be a solution to the equation (III.43). There is $\varepsilon_{0}>0$ such that if $\|\nabla S\|_{L^{2}(\mathbb{C})} \leq \varepsilon_{0}$, then $u \equiv 0$.

Proof of theorem IV.8 From Lemma $\llbracket .3$ we can find $v \in L^{2}\left(\mathbb{C}, \mathbb{R}^{n}\right)$ such that $\nabla^{\perp} v=$ $S \nabla u$.

Assume that $\|\nabla S\|_{L^{2}(\mathbb{C})} \leq \varepsilon_{0}$ where $\varepsilon_{0}$ is the constant appearing in Theorem III.6, Then there is $Q \in \dot{W}^{1,2}(\mathbb{C}, S O(n))$ such that

$$
S=Q^{-1} S^{0} Q
$$

where $S^{0}$ is the matrix (III.47) and $\|\nabla Q\|_{L^{2}(\mathbb{C})} \lesssim \varepsilon_{0}$. 
We set

$$
f=f_{\Re}+i f_{\Im}=S^{0} Q u+i Q v .
$$

Equation (III.67) is equivalent to the system:

$$
\left\{\begin{array}{c}
\partial_{x_{1}} f_{\Re}+\partial_{x_{2}} f_{\Im}=S^{0} \partial_{x_{1}} Q Q^{-1} S^{0} f_{\Re}+\partial_{x_{2}} Q Q^{-1} f_{\Im} \\
-\partial_{x_{2}} f_{\Re}+\partial_{x_{1}} f_{\Im}=-S^{0} \partial_{x_{2}} Q Q^{-1} S^{0} f_{\Re}+\partial_{x_{1}} Q Q^{-1} f_{\Im} .
\end{array}\right.
$$

We can write

$$
S^{0}=\left((-1)^{\min (2 m+1,2 i)} \delta_{i j}\right)_{1 \leq i, j \leq n} .
$$

Let $\Omega=\left(\omega_{i j}\right)_{1 \leq i, j \leq n}$ be an anti-symmetric real matrix (i.e. $\omega_{i j}=-\omega_{j i}$ ), then

$$
\tilde{\Omega}=S^{0} \Omega S^{0}=\left(\omega_{i j}(-1)^{\min (2 m+1,2 i)+\min (2 m+1,2 j)}\right) .
$$

Therefore

$$
\left\{\begin{array}{c}
\tilde{\omega}_{i j}=\omega_{i j} \Longleftrightarrow i, j \leq m \text { and } i, j>m \\
\tilde{\omega}_{i j}=-\omega_{i j} \quad \Longleftrightarrow \text { otherwise }
\end{array}\right.
$$

Observe that the matrix $\tilde{\Omega}$ is still anti-symmetric. We set $\Omega^{\ell}:=\partial_{x_{\ell}} Q Q^{-1}$ and $\tilde{\Omega}^{\ell}=$ $S^{0} \partial_{x_{\ell}} Q Q^{-1} S^{0}$.

$$
\left\{\begin{array}{l}
\partial_{x_{1}} f_{\Re}+\partial_{x_{2}} f_{\Im}=\tilde{\Omega}^{1} f_{\Re}+\Omega^{2} f_{\Im} \\
\partial_{x_{2}} f_{\Re}-\partial_{x_{1}} f_{\Im}=\tilde{\Omega}^{2} f_{\Re}-\Omega^{1} f_{\Im}
\end{array}\right.
$$

Then we get

$$
\begin{aligned}
\left(\partial_{x_{1}}-i \partial_{x_{2}}\right)\left(f_{\Re}+i f_{\Im}\right) & =\tilde{\Omega}^{1} f_{\Re}+\Omega^{2} f_{\Im}-i\left(\tilde{\Omega}^{2} f_{\Re}-\Omega^{1} f_{\Im}\right) \\
& =\left(\tilde{\Omega}^{1}-i \tilde{\Omega}^{2}\right) f_{\Re}+i\left(\Omega^{1}-i \Omega^{2}\right) f_{\Im} \\
& =\left(\frac{\tilde{\Omega}^{1}-i \tilde{\Omega}^{2}}{2}\right)\left(\left(f_{\Re}+i f_{\Im}\right)+\left(f_{\Re}-i f_{\Im}\right)\right) \\
& +\left(\frac{\Omega^{1}-i \Omega^{2}}{2}\right)\left(\left(f_{\Re}+i f_{\Im}\right)-\left(f_{\Re}-i f_{\Im}\right)\right) .
\end{aligned}
$$

Which gives

$$
\begin{aligned}
\left(\partial_{x_{1}}-i \partial_{x_{2}}\right)\left(f_{\Re}+i f_{\Im}\right)= & \frac{1}{2}\left[\left(\tilde{\Omega}^{1}+\Omega^{1}\right)-i\left(\tilde{\Omega}^{2}+\Omega^{2}\right)\right]\left(f_{\Re}+i f_{\Im}\right) \\
& +\frac{1}{2}\left[\left(\tilde{\Omega}^{1}-\Omega^{1}\right)-i\left(\tilde{\Omega}^{2}-\Omega^{2}\right)\right]\left(f_{\Re}-i f_{\Im}\right) .
\end{aligned}
$$


From (IV.151) it follows for $\ell=1,2$

$$
\frac{\tilde{\Omega}^{\ell}+\Omega^{\ell}}{2}=\left(\begin{array}{c|c}
\omega_{i j}^{\ell} & 0_{m \times n-m} \\
\hline 0_{n-m \times m} & \omega_{i j}^{\ell}
\end{array}\right)
$$

and

$$
\frac{\tilde{\Omega}^{\ell}-\Omega^{\ell}}{2}=\left(\begin{array}{c|c}
0_{m \times m} & -\omega_{i j}^{\ell} \\
\hline-\omega_{i j}^{\ell} & 0_{n-m \times n-m}
\end{array}\right) .
$$

We can write the system (IV.154) as

$$
\partial_{L} f=\frac{1}{2} \Omega^{+} f+\frac{1}{2} \Omega^{-} \bar{f}
$$

where

$$
\begin{aligned}
& \Omega^{+}=\frac{\left(\tilde{\Omega}^{1}+\Omega^{1}\right)-i\left(\tilde{\Omega}^{2}+\Omega^{2}\right)}{2} \\
& \Omega^{-}=\frac{\left(\tilde{\Omega}^{1}-\Omega^{1}\right)-i\left(\tilde{\Omega}^{2}-\Omega^{2}\right)}{2} .
\end{aligned}
$$

We observe that by construction for every $i, j$ we have

$$
\begin{aligned}
& \Im\left(\partial_{\bar{z}} \Omega_{i j}^{+}\right)=\partial_{x_{2}}\left(\Omega_{i j}^{+}\right)^{\Re}+\partial_{x_{1}}\left(\Omega_{i j}^{+}\right)^{\Im} \in \mathcal{H}^{1}\left(\mathbb{R}^{2}\right) \\
& \Im\left(\partial_{\bar{z}} \Omega_{i j}^{-}\right)=\partial_{x_{2}}\left(\Omega_{i j}^{-}\right)^{\Re}+\partial_{x_{1}}\left(\Omega_{i j}^{-}\right)^{\Im} \in \mathcal{H}^{1}\left(\mathbb{R}^{2}\right)
\end{aligned}
$$

with

$$
\left\|\Im\left(\partial_{\bar{z}} \Omega_{i j}^{+}\right)\right\|_{\mathcal{H}^{1}\left(\mathbb{R}^{2}\right)} \lesssim\|\nabla Q\|_{L^{2}\left(\mathbb{R}^{2}\right)}^{2},\left\|\Im\left(\partial_{\bar{z}} \Omega_{i j}^{-}\right)\right\| \lesssim\|\nabla Q\|_{L^{2}\left(\mathbb{R}^{2}\right)}^{2}
$$

since these quantities are linear combinations of Jacobians of functions (the components of the matrix $Q$ ) with gradient in $L^{2}$.

Let $M$ be defined as follows:

$$
M=\left(\begin{array}{c|c}
M_{R} & 0_{m \times n-m} \\
\hline 0_{n-m \times m} & M_{L}
\end{array}\right)
$$

where $M_{R} \in \dot{W}^{1,2}\left(\mathbb{R}^{2}, S O(m)\right)$ and $M_{L} \in \dot{W}^{1,2}\left(\mathbb{R}^{2}, S O(n-m)\right)$. The following identity holds

$$
\begin{aligned}
\partial_{L}(M f) & =\partial_{L} M f+M \partial_{z} f=\left(\partial_{L} M M^{-1}\right)(M f)+\frac{1}{2} M\left(\Omega^{+} f+\Omega^{-} \bar{f}\right) \\
& =\left(\partial_{L} M M^{-1}+\frac{1}{2} M \Omega^{+} M^{-1}\right) M f+\frac{1}{2}\left(M \Omega^{-} M^{-1}\right) \overline{M f}
\end{aligned}
$$


Claim 1: There are two constants $\varepsilon(n)>0$ and $C(n)>0$ depending only on $n$ such that if $\left\|\Omega^{+}\right\|_{L^{2}}<\varepsilon(n)$ there exists a matrix $M$ of the form (IV.162) and $\eta \in \dot{W}^{1,(2,1)}\left(\mathbb{R}^{2}\right) \cap L^{\infty}\left(\mathbb{R}^{2}\right)$ such that

$$
\partial_{L} M M^{-1}+\frac{1}{2} M \Omega^{+} M^{-1}=-i \partial_{z} \eta
$$

an

$$
\|\nabla M\|_{L^{2}\left(\mathbb{R}^{2}\right)},\|\nabla \eta\|_{L^{2,1}\left(\mathbb{R}^{2}\right)} \lesssim\left\|\Omega^{+}\right\|_{L^{2}}
$$

Proof of Claim 1. By the same arguments in Lemma A.3 in [14] we can find $M \in$ $\dot{W}^{1,2}(\mathbb{C}, S O(n))$ of the form (IV.162) with $\|\nabla M\|_{L^{2}\left(\mathbb{R}^{2}\right)} \lesssim\left\|\Omega^{+}\right\|_{L^{2}}$ and $\eta \in \dot{W}^{1,2}(\mathbb{C}$, so $(n))$ such that

$$
\left\{\begin{array}{l}
-\partial_{x_{2}} \eta=\partial_{x_{1}} M M^{-1}+M\left[\tilde{\Omega}_{1}+\Omega_{1}\right] M^{-1} \\
\partial_{x_{1}} \eta=\partial_{x_{2}} M M^{-1}+M\left[\tilde{\Omega}_{2}+\Omega_{2}\right] M^{-1}
\end{array}\right.
$$

It follows that

$$
\begin{aligned}
-\Delta \eta & =\underbrace{\partial_{x_{2}}\left(\partial_{x_{1}} M M^{-1}\right)-\partial_{x_{1}}\left(\partial_{x_{2}} M M^{-1}\right)}_{(1)} \\
& +\underbrace{\partial_{x_{2}}\left(M\left(\tilde{\Omega}_{1}+\Omega_{1}\right) M^{-1}\right)-\partial_{x_{1}}\left(M\left(\tilde{\Omega}_{2}+\Omega_{2}\right) M^{-1}\right)}_{(2)} .
\end{aligned}
$$

The first term (1) on the right hand side of (IV.165) is in the Hardy Space $\mathcal{H}^{1}\left(\mathbb{R}^{2}\right)$ since it is a linear combination of Jacobians of functions with derivative in $L^{2}$.

Claim 2: The second term $(2)$ is in $\dot{W}^{-1,(2,1)}\left(\mathbb{R}^{2}\right)$.

Proof of the Claim 2. Indeed we observe that each component of (2) can be written in the form

$$
\partial_{x_{2}}\left(a \omega_{1}\right)-\partial_{x_{1}}\left(a \omega_{2}\right)
$$

where $a \in\left(\dot{W}^{1,2} \cap L^{\infty}\right)\left(\mathbb{R}^{2}\right)$ and $\omega_{1}, \omega_{2} \in L^{2}\left(\mathbb{R}^{2}\right)$ satisfy

$$
\partial_{x_{2}} \omega_{1}-\partial_{x_{1}} \omega_{2} \in \mathcal{H}^{1}\left(\mathbb{R}^{2}\right)
$$

Actually we have

$$
\begin{aligned}
& \partial_{x_{2}}\left(M\left(\tilde{\Omega}_{1}+\Omega_{1}\right) M^{-1}\right)_{i j}-\partial_{x_{1}}\left(M\left(\tilde{\Omega}_{2}+\Omega_{2}\right) M^{-1}\right)_{i j} \\
= & \partial_{x_{2}}\left(M_{i k}\left(\tilde{\Omega}_{1}+\Omega_{1}\right)_{k t} M_{t j}^{-1}\right)-\partial_{x_{1}}\left(M_{i k}\left(\tilde{\Omega}_{2}+\Omega_{2}\right)_{k t} M_{t j}^{-1}\right) .
\end{aligned}
$$

One sets

$$
a=M_{i k} M_{t j}^{-1}, \quad \omega_{1}=\left(\tilde{\Omega}_{1}+\Omega_{1}\right)_{k t}, \quad \omega_{2}=\left(\tilde{\Omega}_{2}+\Omega_{2}\right)_{k t} .
$$


Let $c, b \in \dot{W}^{1,2}\left(\mathbb{R}^{2}\right)$ be such that

$$
\left(\begin{array}{c}
\omega_{1} \\
\omega_{2}
\end{array}\right)=\nabla^{\perp} c+\nabla b .
$$

We can deduce from (IV.168) that $\Delta c \in \mathcal{H}^{1}\left(\mathbb{R}^{2}\right)$ hence $c \in \dot{W}^{1,(2,1)}\left(\mathbb{R}^{2}\right)$. We can now rewrite (IV.167) as follows

$$
\begin{aligned}
\partial_{x_{2}}\left[a\left(\partial_{x_{1}} b-\partial_{x_{2}} c\right)\right]-\partial_{x_{1}}\left[a\left(\partial_{x_{2}} b+\partial_{x_{1}} c\right)\right] & =\partial_{x_{2}} a \partial_{x_{1}} b-\partial_{x_{1}} a \partial_{x_{2}} b \\
& -\left(\partial_{x_{1}}\left[a \partial_{x_{1}} c\right]+\partial_{x_{2}}\left[a \partial_{x_{2}} c\right]\right)
\end{aligned}
$$

We observe that $\partial_{x_{2}} a \partial_{x_{1}} b-\partial_{x_{1}} a \partial_{x_{2}} b \in \mathcal{H}^{1}\left(\mathbb{R}^{2}\right)$ and $\partial_{x_{1}}\left[a \partial_{x_{1}} c\right]+\partial_{x_{2}}\left[a \partial_{x_{2}} c\right] \in \dot{W}^{-1,(2,1)}\left(\mathbb{R}^{2}\right)$. This gives that $(2)$ is in $\dot{W}^{-1,(2,1)}\left(\mathbb{R}^{2}\right)$ and this concludes the proof of Claim 1 and Claim 2.

The system (IV.163) can then be written as

$$
\partial_{L}(M f)=A(M f)+B \overline{M f}
$$

with $A=-i \partial_{z} \eta \in L^{2,1}\left(\mathbb{R}^{2}, \mathcal{M}_{n \times n}(\mathbb{C})\right)$ and $B=\frac{1}{2}\left(M \Omega^{-} M^{-1}\right) \in L^{2}\left(\mathbb{R}^{2}, \mathcal{M}_{n \times n}(\mathbb{C})\right)$ with

$$
\|\nabla A\|_{L^{2,1}\left(\mathbb{R}^{2}\right)},\|\nabla B\|_{L^{2}\left(\mathbb{R}^{2}\right)} \lesssim\|\nabla M\|_{L^{2}}^{2}
$$

$B$ satisfies for every $i, j B_{i j}=-B_{j i}$ and

$$
\partial_{x_{2}}\left(B_{i j}^{\Re}\right)+\partial_{x_{1}}\left(B_{i j}^{\Im}\right) \in \dot{W}^{-1,(2,1)}\left(\mathbb{R}^{2}\right) .
$$

Proof of the Claim 3. For every ij we have $B_{i j}=\frac{1}{2}\left(M_{i k} \Omega_{k t}^{-} M_{t j}^{-1}\right)$. We know from (IV.161) that

$$
\begin{gathered}
\Im\left(\partial_{\bar{z}} \Omega_{k t}^{-}\right)=\partial_{x_{2}}\left(\Omega_{k t}^{-}\right)^{\Re}+\partial_{x_{1}}\left(\Omega_{k t}^{-}\right)^{\Im} \in \mathcal{H}^{1}\left(\mathbb{R}^{2}\right) \\
\left\|\Im\left(\partial_{\bar{z}} \Omega_{k t}^{-}\right)\right\|_{\mathcal{H}^{1}\left(\mathbb{R}^{2}\right)} \lesssim\|\nabla Q\|_{L^{2}\left(\mathbb{R}^{2}\right)}^{2}
\end{gathered}
$$

We proceed as in the proof of Claim 2: let $c, b \in \dot{W}^{1,2}\left(\mathbb{R}^{2}\right)$ be such that

$$
\left(\begin{array}{c}
\left(\Omega_{k t}^{-}\right)^{\Re} \\
-\left(\Omega_{k t}^{-}\right)^{\Im}
\end{array}\right)=\nabla^{\perp} c+\nabla b .
$$

We can deduce from (IV.174) that $\Delta c \in \mathcal{H}^{1}\left(\mathbb{R}^{2}\right)$ hence $c \in \dot{W}^{1,(2,1)}\left(\mathbb{R}^{2}\right)$. Then setting $a=M_{i k} M_{t j}^{-1}$ we have

$$
\begin{aligned}
& \left.\partial_{x_{2}}\left(a \Omega_{k t}^{-}\right)^{\Re}\right)+\partial_{x_{1}}\left(a \Omega_{k t}^{-}\right)^{\Im}=\partial_{x_{2}}\left(a\left(\partial_{x_{1}} b-\partial_{x_{2}} c\right)+\partial_{x_{1}}\left(a\left(-\partial_{x_{2}} b-\partial_{x_{1}} c\right)\right)\right. \\
= & \partial_{x_{2}} a \partial_{x_{1}} b-\partial_{x_{1}} a \partial_{x_{2}} b-\left(\partial_{x_{2}}\left(a \partial_{x_{2}} c\right)+\partial_{x_{1}}\left(a \partial_{x_{1}} c\right) .\right.
\end{aligned}
$$


We then conclude as in the proof of Claim 3.

In the sequel we can focus our attention to a system of the type:

$$
\partial_{L} g=A g+B \bar{g}
$$

where $A \in L^{2,1}\left(\mathbb{R}^{2}, \mathcal{M}_{n \times n}(\mathbb{C})\right)$ and $B \in L^{2}\left(\mathbb{R}^{2}, \mathcal{M}_{n \times n}(\mathbb{C})\right)$ satisfying $B_{i j}=-B_{j i}$ and (IV.172)

Step 1. We first observe that

$$
\partial_{L} g=A g-B j g j
$$

where $j$ is the quaternion number satisfying $j^{2}=-1$ and $i j=-j i$.

Step 2. The function $g j$ satisfies the system

$$
\partial_{L} g j=A g j+B j g .
$$

Step 3. We set

$$
G=\left(\begin{array}{c}
g^{1} \\
\vdots \\
g^{n} \\
g^{1} j \\
\vdots \\
g^{n} j
\end{array}\right)
$$

$G$ satisfies

$$
\partial_{L} G=\Gamma G+\Gamma_{1} G,
$$

where

$$
\Gamma_{1}=\Gamma_{1}^{\Re}-i \Gamma_{1}^{\Im}=\left(\begin{array}{c|c}
A & 0_{n \times n} \\
\hline 0_{n \times n} & A
\end{array}\right),
$$

and

$$
\Gamma=\Gamma^{\Re}+i \Gamma^{\Im}=\left(\begin{array}{c|c}
0_{n \times n} & -B j \\
\hline B j & 0_{n \times n}
\end{array}\right),
$$

where we have set $B=B^{\Re}+i B^{\Im}$ and

$$
\Gamma^{\Re}:=\left(\begin{array}{c|c}
0_{n \times n} & (-B)^{\Re} j \\
\hline B^{\Re} j & 0_{n \times n}
\end{array}\right) \quad \text { and } \quad \Gamma^{\Im}:=\left(\begin{array}{c|c}
0_{n \times n} & (-B)^{\Im} j \\
\hline B^{\Im} j & 0_{n \times n}
\end{array}\right) .
$$


Observe that

$$
\overline{\Gamma^{\Re}}:=\left(\begin{array}{c|c}
0_{n \times n} & B^{\Re} j \\
\hline-B^{\Re} j & 0_{n \times n}
\end{array}\right)
$$

and then

$$
\left(\overline{\Gamma^{\Re}}\right)^{t}=\left(\begin{array}{c|c}
0_{n \times n} & -\left(B^{t}\right)^{\Re} j \\
\hline\left(B^{t}\right)^{\Re} j & 0_{n \times n}
\end{array}\right)=-\Gamma^{\Re}
$$

Therefore

$$
\left(\overline{\Gamma^{\Re}}\right)^{t}+\Gamma^{\Re}=0 .
$$

Similarly $\left(\overline{\Gamma^{\Im}}\right)^{t}+\Gamma^{\Im}=0$.

Since the coefficients of $\Gamma^{\Im}$ are in $j \mathbb{R}$, we obtain $\overline{i \Gamma^{\Im}}=-\overline{\Gamma^{\Im}} i=i \overline{\Gamma^{\Im}}$. Hence finally we have established

$$
\left(\overline{i \Gamma^{\Im}}\right)^{t}=i\left(\overline{\Gamma^{\Im}}\right)^{t}=-i \Gamma^{\Im}
$$

The matrix $\Gamma=\Gamma^{\Re}+i \Gamma^{\Im}$ satisfies then $(\bar{\Gamma})^{t}+\Gamma=0$ which means that it belongs to the Lie algebra $u(2 n, \mathbb{H})$ of the hyper-unitary group $U(2 n, \mathbb{H})$. This is the compact Lie group of invertible $2 n \times 2 n$ quaternions matrices $D$ satisfying $\bar{D}^{t} D=D \bar{D}^{t}=I d_{n}$.

Let $G$ be a $L^{2}$ solution of (IV.180) with $\Gamma \in L^{2}\left(\mathbb{R}^{2}, u(2 n, \mathbb{H})\right), \Gamma_{1} \in L^{2,1}\left(\mathbb{R}^{2}, \mathcal{M}_{2 n \times 2 n}(\mathbb{H})\right)$. Let us take $P \in L^{2}\left(\mathbb{R}^{2}, U(n, \mathbb{H})\right.$ ) (to be fixed later), then the following estimates hold

$$
\begin{aligned}
& \partial_{x_{1}}(P G)-\partial_{x_{2}}(P i G)=P\left[\left(\partial_{x_{1}} G-i \partial_{x_{2}} G\right)+P^{-1} \partial_{x_{1}} P-P^{-1} \partial_{x_{2}} P i\right] G \\
= & P\left[2\left(\Gamma_{1}+\Gamma\right)+\left(P^{-1} \partial_{x_{1}} P-P^{-1} \partial_{x_{2}} P i\right)\right] P^{-1}(P G) .
\end{aligned}
$$

The key point is to choose $P$ in order to absorb in (IV.184) the term $2 \Gamma$.

We first observe that if $P \in U(2 n, \mathbb{H})$ then $P^{-1} \nabla P \in u(2 n, \mathbb{H})$. Actually since $P^{-1}=$ $\bar{P}^{t}$ and $P^{-1} \nabla P=-\nabla P^{-1} P$ one has

$$
{\overline{\left(P^{-1} \nabla P\right)}}^{t}=\left(\nabla \bar{P}^{t}\right){\overline{P^{-1}}}^{t}=\nabla P^{-1} P=-P^{-1} \nabla P .
$$

10 We also recall that every matrix $U \in u(2 n, \mathbb{H})$ can be represented as

$$
U=U_{0}+U_{1} i+U_{2} j+U_{3} k,
$$

\footnotetext{
${ }^{10}$ We recall that the standard Hermitian form in $\mathbb{H}^{n}$ is defined by $\langle x, y\rangle:=\sum_{i=1}^{n} \bar{x}_{i} y_{i}$. Therefore given $A, B$ two $n \times n$ matrices with entries in $\mathbb{H}$ we have

$$
\langle A B x, y\rangle=\left\langle B x, \bar{A}^{t} y\right\rangle=\left\langle x, \bar{B}^{t} \bar{A}^{t} y\right\rangle
$$

and therefore $\overline{(A B)}^{t}=\bar{B}^{t} \bar{A}^{t}$, (see e.g. [23], Section 4).
} 
where $U_{i}$ are real $2 n \times 2 n$ matrices such that $U_{0}^{t}=-U_{0}$ and $U_{i}^{t}=U_{i}$. for $i=1,2,3$.

Now we are going to proceed as in Section III.2.

In the sequel we will denote by $\operatorname{MSpan}\{1, i\}$ the space of $n \times n$ matrices $A+i B$ where $A, B$ a real-valued $2 n \times 2 n$ matrices, with $A^{t}=-A$ and $B^{t}=B$ and $\operatorname{MSpan}\{j, k\}$ will denote the space of $2 n \times 2 n$ matrices $j C+k D$ where $C, D$ a real-valued $2 n \times 2 n$ matrices, with $C^{t}=C$ and $D^{t}=D$.

We are going to show first an analogous of Theorem $\llbracket 11.7$.

Theorem IV.9. There exists $\varepsilon_{0}>0$ such that for every $\Gamma \in \dot{W}^{1,2}(\mathbb{C}, M \operatorname{Span}\{1, i\})$ satisfying $\|\Gamma\|_{L^{2}} \leq \varepsilon_{0}$, every $\Gamma_{1} \in \dot{W}^{1,(2,1)}\left(\mathbb{C}, \mathcal{M}_{2 n \times 2 n}(\mathbb{H})\right)$ with $\left\|\Gamma_{1}\right\|_{L^{2,1}} \leq \varepsilon_{0}$ and every $G \in L^{2}(\mathbb{C}, \mathbb{H})$ solving

$$
\partial_{L} G=\left(\Gamma+\Gamma_{1}\right) G
$$

then $G \equiv 0$.

As in the case of $2 \mathrm{D}$ codomains the key step to prove Theorem IV.9 is the following result.

Proposition IV.5. Let $G \in L^{2}\left(\mathbb{C}, \mathbb{H}^{2 n}\right)$ be a solution of (IV.185). There exists an $\varepsilon_{0}>0$ such that if $\|\Gamma\|_{L^{2}} \leq \varepsilon_{0}$ and $\left\|\Gamma_{1}\right\|_{L^{2,1}} \leq \varepsilon_{0}$, then there is a $P \in \dot{W}^{1,2}\left(\mathbb{R}^{2}, u(2 n, \mathbb{H})\right)$ and $\chi \in \dot{W}^{1,(2,1)}\left(\mathbb{R}^{2}, M \operatorname{Span}\{1, i\}\right)$ such that $\|\nabla \chi\|_{L^{2,1}} \leq \varepsilon_{0}$ and

$$
\partial_{x_{1}}(P G)-\partial_{x_{2}}(P i G)=P\left(-\partial_{x_{2}} \chi-\partial_{x_{1}} \chi i+2 \Gamma_{1}\right) G=2 P\left(-i \partial_{L} \chi+\Gamma_{1}\right) G .
$$

Proof of Proposition IV.5. If $G$ solves (IV.185) then as we have seen in (IV.184) for every $P \in u(2 n, \mathbb{H})$ we have

$$
\partial_{x_{1}}(P G)-\partial_{x_{2}}(P i G)=P\left[2 \Gamma+2 \Gamma_{1}+\left(P^{-1} \partial_{x_{1}} P-P^{-1} \partial_{x_{2}} P i\right)\right] G
$$

Step 1. We introduce the following operator

$$
\begin{aligned}
\mathbf{N}: & \dot{W}^{1,2}(\mathbb{C}, U(2 n, \mathbb{H})) \rightarrow \dot{W}^{-1,2}(\mathbb{C}, \operatorname{MSpan}\{1, i\}) \times L^{2}(\mathbb{C}, \operatorname{MSpan}\{j, k\}) \\
P & \mapsto\left(\Pi_{1 i}\left(\partial_{x_{1}}\left(P^{-1} \partial_{x_{1}} P\right)+\partial_{x_{2}}\left(P^{-1} \partial_{x_{2}} P\right)\right), \Pi_{j k}\left(P^{-1} \partial_{x_{1}} P-P^{-1} \partial_{x_{2}} P i\right)\right.
\end{aligned}
$$

Claim 1: $\mathbf{N}$ satisfies the following property: there is $\varepsilon_{0}>0$ and $C>0$ such that for any choice of $V \in \dot{W}^{-1,2}(\mathbb{C}, \operatorname{MSpan}\{1, i\})$ and $T \in L^{2}(\mathbb{C}, \operatorname{MSpan}\{j, k\})$ satisfying

$$
\|V\|_{\dot{W}^{-1,2}} \leq \varepsilon_{0}, \quad\|T\|_{L^{2}} \leq \varepsilon_{0},
$$

then there is $P \in \dot{W}^{1,2}(\mathbb{C}, U(2 n, \mathbb{H}))$ with

$$
\mathbf{N}(P)=(V, T)
$$

and

$$
\|\nabla P\|_{L^{2}} \leq C\left(\|V\|_{\dot{W}^{-1,2}}+\|\left. T\right|_{L^{2}}\right) .
$$


Proof of Claim 1. The proof of Claim 1 is very similar to that of Lemma III.5, therefore we will sketch only the main arguments. For every $P_{0} \in U(n, \mathbb{H})$ we consider perturbations of the type: $P=P_{0} e^{t U}$ where $U \in u(2 n, \mathbb{H})$ and we set $\tilde{\mathbf{N}}_{P_{0}}(U)=\mathbf{N}\left(P_{0} e^{U}\right)$ and

We have

$$
D \tilde{N}_{P_{0}}(0)=\left.\frac{d}{d t} \tilde{N}_{P_{0}}(t U)\right|_{t=0}=: L_{P_{0}}(U)
$$

$$
\begin{array}{r}
L_{P_{0}}(U):=\left(\Pi_{1 i}\left(\Delta U+\partial_{x_{1}}\left[P_{0}^{-1} \partial_{x_{1}} P_{0} U-U P_{0}^{-1} \partial_{x_{1}} P_{0}\right]+\partial_{x_{2}}\left[P_{0}^{-1} \partial_{x_{2}} P_{0} U-U P_{0}^{-1} \partial_{x_{2}} P_{0}\right]\right),\right. \\
\left.\Pi_{j k}\left(\partial_{x_{1}} U-\partial_{x_{2}} U i+\left[P_{0}^{-1} \partial_{x_{1}} P_{0} U-U P_{0}^{-1} \partial_{x_{1}} P_{0}\right]-\left[P_{0}^{-1} \partial_{x_{2}} P_{0} U-U P_{0}^{-1} \partial_{x_{2}} P_{0}\right] i\right)\right) .
\end{array}
$$

In the case $P_{0}=I d$ we get

$$
\begin{aligned}
L_{I d}(U) & :=\left(\Pi_{1 i}(\Delta U), \Pi_{j k}\left(\partial_{x_{1}} U-\partial_{x_{2}} U i\right)\right) \\
& =\left(\Delta\left(U_{0}+i U_{1}\right),\left(\partial_{x_{1}} U_{2}-\partial_{x_{2}} U_{3}\right) j+\left(\partial_{x_{1}} U_{3}+\partial_{x_{2}} U_{2}\right) k\right) .
\end{aligned}
$$

Now by arguing exactly as in the proof of Theorem III.7 one can prove that if $\varepsilon_{0}$ in (IV.188) is small enough then $L_{P_{0}}$ with $\left\|\nabla P_{0}\right\|_{L^{2}}<\varepsilon_{0}$ is invertible, therefore the Claim 1 holds.

Step 2. From Step 1 it follows that if $\|\Gamma\|_{L^{2}}<\varepsilon_{0}$ then there is $P \in \dot{W}^{1,2}(\mathbb{C}, U(2 n, \mathbb{H}))$ such that

$$
\left\{\begin{array}{c}
\Pi_{1 i}\left(\partial_{x_{1}}\left(P^{-1} \partial_{x_{1}} P\right)+\partial_{x_{2}}\left(P^{-1} \partial_{x_{2}} P\right)\right)=0 \\
\Pi_{j k}\left(P^{-1} \partial_{x_{1}} P-P^{-1} \partial_{x_{2}} P i\right)=-2 \Gamma .
\end{array}\right.
$$

From the first equation in (IV.191) it follows the existence of $\chi \in \dot{W}^{1,2}(\mathbb{C}, \operatorname{MSpan}\{1, i\})$ such that

$$
\left\{\begin{array}{l}
\Pi_{1 i}\left(P^{-1} \partial_{x_{1}} P\right)=-\partial_{x_{2}} \chi \\
\Pi_{1 i}\left(P^{-1} \partial_{x_{2}} P\right)=\partial_{x_{1}} \chi
\end{array}\right.
$$

From (IV.192) it follows in particular that

$$
\begin{aligned}
-\Delta \chi & =\partial_{x_{2}}\left(\Pi_{1 i}\left(P^{-1} \partial_{x_{1}} P\right)\right)-\partial_{x_{1}}\left(\Pi_{1 i}\left(P^{-1} \partial_{x_{2}} P\right)\right) \\
& =\Pi_{1 i}\left(\partial_{x_{2}}\left(P^{-1} \partial_{x_{1}} P\right)-\partial_{x_{1}}\left(P^{-1} \partial_{x_{2}} P\right)\right) .
\end{aligned}
$$

The right hand side of (III.124) is a sum of Jacobians, hence it is in the Hardy space $\mathcal{H}^{1}(\mathbb{C})$. This implies that $\nabla \chi \in L^{2,1}(\mathbb{C})$, with $\|\nabla \chi\|_{L^{2,1}} \lesssim\|\nabla P\|_{L^{2}}^{2}$.

We have

$$
\begin{aligned}
P^{-1} \partial_{x_{1}} P-P^{-1} \partial_{x_{2}} P i & =\Pi_{1 i}\left(P^{-1} \partial_{x_{1}} P\right)+\Pi_{j k}\left(P^{-1} \partial_{x_{1}} P\right)-\Pi_{1 i}\left(P^{-1} \partial_{x_{2}} P\right) i-\Pi_{j k}\left(P^{-1} \partial_{x_{2}} P\right) i \\
& =-\partial_{x_{2}} \chi-\partial_{x_{1}} \chi i+\Pi_{j k}\left(P^{-1} \partial_{x_{1}} P-P^{-1} \partial_{x_{2}} P i\right) \\
& =-\partial_{x_{2}} \chi-\partial_{x_{1}} \chi i-2 \Gamma .
\end{aligned}
$$


By combining (IV.187) (IV.192) and (IV.194) we get

$$
\partial_{x_{1}}(P G)-\partial_{x_{2}}(P i G)=P\left(2 \Gamma_{1}-\partial_{x_{2}} \chi-\partial_{x_{1}} \chi i\right) G=2 P\left(-i \partial_{L} \chi+\Gamma_{1}\right) G
$$

and we conclude the proof of the proposition IV.5.

Proof of Theorem IV.9. By arguing as in the end of the proof of Theorem III.7 from (IV.195) we deduce that

$$
\begin{aligned}
\|P G\|_{L^{2, \infty}} & \lesssim\left(\|\nabla \chi\|_{L^{2,1}}+\left\|\Gamma_{1}\right\|_{L^{2,1}}\right)\|P G\|_{L^{2, \infty}} \\
& \lesssim \varepsilon_{0}\|P G\|_{L^{2, \infty}} .
\end{aligned}
$$

If $\varepsilon_{0}$ is small enough then $G \equiv 0$. This concludes the proof of theorem IV.9 and therefore of theorem IV.8

From theorem IV.9 it follows theorem I.1 in the general case $n \geq 2$. The proof is the same of that of Proposition III.4 and therefore we omit it.

\section{Proof of theorem $\underline{I} .2$}

A standard covering argument gives that, modulo extraction of a subsequence, there exist finitely many points $a_{1} \ldots a_{Q}$ such that, for any $\delta>0$

$$
\lim _{k \rightarrow+\infty} \inf \left\{\rho>0 ; \int_{B_{\rho}(x)}\left|\nabla S_{k}\right|^{2}(y) d y=\frac{\varepsilon_{0}^{2}}{2} \text { where } x \in \mathbb{R}^{2} \backslash \cup_{i=1}^{Q} B_{\delta}\left(a_{i}\right)\right\}>0
$$

where $\varepsilon_{0}>$ is given by the epsilon-regularity theorem IV.8. Theorem I.1 implies then that $u_{k} \rightarrow u_{\infty}$ strongly in $L_{l o c}^{2}\left(\mathbb{R}^{2} \backslash\left\{a_{1} \ldots a_{Q}\right\}\right)$ hence we can pass in the limit in the equation away from the points and one gets

$$
\operatorname{div}\left(S_{\infty} \nabla u_{\infty}\right)=0 \quad \text { in } \mathcal{D}^{\prime}\left(\mathbb{R}^{2} \backslash\left\{a_{1} \ldots a_{Q}\right\}\right) .
$$

It remains to establish the point removability. Since $S_{\infty} \nabla u_{\infty}=\nabla\left(S_{\infty} u_{\infty}\right)-\nabla S_{\infty} u_{\infty} \in$ $W^{-1,2}+L^{1}\left(\mathbb{R}^{2}\right)$ a classical result on distributions supported by points gives the existence of $\alpha_{1} \ldots \alpha_{Q} \in \mathbb{R}^{n}$ such that

$$
\operatorname{div}\left(S_{\infty} \nabla u_{\infty}\right)=\sum_{i=1}^{H} \alpha_{i} \delta_{a_{i}} \quad \text { in } \mathcal{D}^{\prime}\left(\mathbb{R}^{2}\right) .
$$

We pick a point $a_{i_{0}}$ arbitrary and we consider an axially symmetric function $\chi$ centered at $a_{i_{0}}$ such that $\chi \equiv 1$ in a neighborhood of $a_{i_{0}}$ and $\operatorname{Supp} \chi \subset B\left(a_{i_{0}}, r\right)$ where $0<r<$ $\inf _{i \neq j}\left|a_{i}-a_{j}\right|$. We have

$$
0=\int_{B\left(a_{i_{0}}, r\right)} \nabla \chi \cdot S_{k} \nabla u_{k} d x
$$


Because of the weak convergence of $\nabla u_{k}$ towards $\nabla u_{\infty}$ in $L^{q}$ for any $q<2$ away from the points $a_{1} \ldots a_{Q}$ and the strong convergence of $S_{k}$ towards $S_{\infty}$ in any $L_{l o c}^{p}$ for $p<+\infty$ we have

$$
0=\int_{B\left(a_{i_{0}}, r\right)} \nabla \chi \cdot S_{\infty} \nabla u_{\infty} d x
$$

which gives $\alpha_{i_{0}}=0$. This concludes the proof of theorem $\llbracket .2$.

\section{References}

[1] Adams, D. R. A note on Riesz potentials. Duke Math. J. 42 (1975), no. 4, 765-778.

[2] Bourgain, J. ; Brezis, H. On the equation $\operatorname{div} Y=f$ and application to control of phases. J. Amer. Math. Soc. 16 (2003), no. 2, 393-426.

[3] Coifman, R. R.; Rochberg, R.; Weiss, Guido Factorization theorems for Hardy spaces in several variables. Ann. of Math. (2) 103 (1976), no. 3, 611-635.

[4] Coifman, R.; Lions, P.-L.; Meyer, Y.; Semmes, S. Compensated compactness and Hardy spaces. J. Math. Pures Appl. (9) 72 (1993), no. 3, 247-286

[5] Da Lio, F.; Rivière, T. Sub-criticality of non-local Schrödinger systems with antisymmetric potentials and applications to half-harmonic maps, Advances in Mathematics 227, (2011), 1300-1348.

[6] Da Lio, F.; Rivière, T. 3-commutators revisited preprint 2019. Nonlinear Anal., 165, 2017, 182-197.

[7] Delort, J.M. Existence de nappes de tourbillon en dimension deux. (French) [Existence of vortex sheets in dimension two] J. Amer. Math. Soc. 4 (1991), no. 3, 553-586.

[8] Evans, L.C. ; Müller, S. Hardy spaces and the two-dimensional Euler equations with nonnegative vorticity. J. Amer. Math. Soc. 7 (1994), no. 1, 199-219.

[9] Grafakos, L. Classical Fourier analysis. Third edition. Graduate Texts in Mathematics, 249. Springer, New York, 2014.

[10] Hélein, F. Harmonic maps, conservation laws and moving frames. Second edition. Cambridge Tracts in Mathematics, 150. Cambridge University Press, Cambridge, 2002.

[11] Hélein, F.; Romon, P. Weierstrass representation of Lagrangian surfaces in fourdimensional space using spinors and quaternions. Comment. Math. Helv. 75 (2000), no. 4, 668-680. 
[12] Jin, T. ; Maz'ya, V. ; Van Schaftingen, J. Pathological solutions to elliptic problems in divergence form with continuous coefficients. C. R. Math. Acad. Sci. Paris 347 (2009), no. 13-14, 773-778.

[13] Maz'ya, V. Bourgain-Brezis type inequality with explicit constants. Interpolation theory and applications, 247-252, Contemp. Math., 445, Amer. Math. Soc., Providence, RI, 2007.

[14] Rivière, T. Conservation laws for conformally invariant variational problems, Invent. Math. 168 (1) (2007) 1-22.

[15] Rivière, T. Sub-criticality of Schrödinger systems with antisymmetric potentials. J. Math. Pures Appl. (9) 95 (2011), no. 3, 260-276.

[16] Rivière, T. Sequences of smooth global isothermic immersions. Comm. Partial Differential Equations 38 (2013), no. 2, 276-303.

[17] Rivière, T. Personal communication.

[18] Schikorra, A. A remark on gauge transformations and the moving frame method. Ann. Inst. H. Poincaré Anal. Non Linéaire 27 (2010), no. 2, 503-515.

[19] Simon, B. Schrödinger semigroups. Bull. Amer. Math. Soc. (N.S.) 7 (1982), no. 3, 447-526.

[20] Stein, E. M. Singular integrals and differentiability properties of functions. Princeton Mathematical Series, No. 30 Princeton University Press, Princeton, N.J. 1970

[21] Uhlenbeck, K. Connections with $L^{p}$ bounds on curvature. Comm. Math. Phys. 83 (1982), no. 1, 31-42.

[22] Wente, H. C. An existence theorem for surfaces of constant mean curvature. J. Math. Anal. Appl. 261969 318-344.

[23] Zhang, F. Quaternions and matrices of quaternions. Linear Algebra and its Applications, Volume 251, 15 January 1997, 21-57. 